\title{
A Review of Doses for Dental Imaging in 2010-2020 and Development of a Web Dose Calculator
}

\author{
Hawon Lee $\mathbb{i D}^{1,2}$ and Andreu Badal $\mathbb{i D}^{2}$ \\ ${ }^{1}$ Montgomery Blair High School, Silver Spring, MD, USA \\ ${ }^{2}$ Division of Imaging, Diagnostics and Software Reliability, OSEL, CDRH, Food and Drug Administration, Silver Spring, \\ MD, USA \\ Correspondence should be addressed to Hawon Lee; gracehawonlee@gmail.com
}

Received 11 April 2021; Revised 26 October 2021; Accepted 27 October 2021; Published 10 December 2021

Academic Editor: André Luiz Ferreira Costa

Copyright (c) 2021 Hawon Lee and Andreu Badal. This is an open access article distributed under the Creative Commons Attribution License, which permits unrestricted use, distribution, and reproduction in any medium, provided the original work is properly cited.

\begin{abstract}
Dental imaging is one of the most common types of diagnostic radiological procedures in modern medicine. We introduce a comprehensive table of organ doses received by patients in dental imaging procedures extracted from literature and a new web application to visualize the summarized dose information. We analyzed articles, published after 2010, from PubMed on organ and effective doses delivered by dental imaging procedures, including intraoral radiography, panoramic radiography, and cone-beam computed tomography (CBCT), and summarized doses by dosimetry method, machine model, patient age, and technical parameters. Mean effective doses delivered by intraoral, $1.32(0.60-2.56) \mu \mathrm{Sv}$, and panoramic, $17.93(3.47-75.00) \mu \mathrm{Sv}$, procedures were found to be about $\%$ and $15 \%$ of that delivered by CBCT, 121.09 (17.10-392.20) $\mu$ Sv, respectively. In CBCT imaging, child phantoms received about $29 \%$ more effective dose than the adult phantoms received. The effective dose of a large field of view (FOV) $\left(>150 \mathrm{~cm}^{2}\right)$ was about 1.6 times greater than that of a small FOV $\left(<50 \mathrm{~cm}^{2}\right)$. The maximum CBCT effective dose with a large FOV for children, $392.2 \mu \mathrm{Sv}$, was about $13 \%$ of theeffective dose that a person receives on average every year from natural radiation, $3110 \mu \mathrm{Sv}$. Monte Carlo simulations of representative cases of the three dental imaging procedures were then conducted to estimate and visualize the dose distribution within the head. The user-friendly interactive web application (available at http:// dentaldose.org) receives user input, such as the number of intraoral radiographs taken, and displays total organ and effective doses, dose distribution maps, and a comparison with other medical and natural sources of radiation. The web dose calculator provides a practical resource for patients interested in understanding the radiation doses delivered by dental imaging procedures.
\end{abstract}

\section{Introduction}

Dental imaging is one of the most common types of diagnostic radiological procedures taken by the average person. Popular dental imaging procedures include intraoral radiography, which has the longest history of use, followed by panoramic radiography, and more recently, cone-beam computed tomography (CBCT) [1]. Intraoral radiography, a simple two-dimensional (2D) projection imaging, is often used to detect periodontal disease and cavities at regular dental check-ups. Panoramic radiography, a more comprehensive $2 \mathrm{D}$ image that combines a series of narrow $2 \mathrm{D}$ images, has been widely used to provide a wide range of information about the dentition and jaws. Introduced in the late 1990s, three-dimensional (3D) imaging technology, CBCT, offers a comprehensive set of cross-sectional images, the ability of vertical scanning, and real-time intraoperative assessment. All three procedures expose different portions of the head, from small parts of the teeth to the whole lower head, to ionizing radiation. There are concerns about the increasing use of imaging procedures as well as the resulting radiation dose, especially for pediatric patients $[2,3]$.

Absorbed dose is defined as the energy deposited to a given volume divided by the mass (measured in gray, Gy, in the International System of Units) [4]. Equivalent dose (measured in sieverts, Sv) is derived from the absorbed dose multiplied by the radiation weighting factor, which represents the effectiveness of the biological damage to the 
exposed tissue. Effective dose (measured in sieverts, Sv) is then derived by adding all equivalent doses multiplied by tissue weighting factors and provides a relative measure of the risk of stochastic effects that might result from irradiation. The most fundamental dose quantity, organ dose, of dental imaging can be obtained through two methods: measurement and computer simulation. First, organ doses can be physically measured with dosimeters placed within anatomy models, called physical human phantoms, that are exposed to dental radiation. Second, organ doses can be calculated through computer simulations where the simulation model of an imaging device is combined with digital anatomy models, called computational human phantoms [5]. Different types of pediatric and adult computational human phantoms are available for dose calculations. Many studies report organ doses from dental imaging procedures estimated by measurement or simulation. However, there are few resources that summarize a variety of data and present the radiation dose with a user-friendly interface.

The current study was intended to provide a practical resource for patients interested in understanding the radiation doses delivered by dental imaging procedures for the period of 2010-2020 and comparison with other radiation sources that are commonly faced in daily life. We established a comprehensive table of organ doses for dental imaging procedures by extracting data from literature and developed a user-friendly web application to present the summarized information.

\section{Materials and Methods}

We obtained articles from PubMed, published after 2010, on organ and effective doses delivered by dental imaging procedures including intraoral radiography, panoramic radiography, and $\mathrm{CBCT}$, and summarized doses by dosimetry method, machine model, patient age, and technical parameters. Monte Carlo simulations of representative cases of the three dental imaging procedures were conducted to estimate and visualize the dose distribution within the head. Finally, we developed an interactive web-based dose calculator to provide easy access to the dental doses and to compare them with other radiation sources commonly faced in daily life.

2.1. Literature Search. We searched for articles on organ and effective doses delivered by dental imaging in PubMed (https://pubmed.ncbi.nlm.nih.gov, National Library of Medicine, National Center for Biotechnology Information) available on October 1, 2020, using the following keywords:
(i) "dental intraoral organ dose" (for intraoral)
(ii) "dental panoramic organ dose" (for panoramic)
(iii) "dental cone beam CT organ dose" (for CBCT)

These keywords brought up 41, 49, and 54 papers (144 in total) for intraoral, panoramic, and CBCT procedures, respectively. We selected papers published after 2010:a total of 81 papers $(14,20$, and 47 papers for intraoral, panoramic, and CBCT, respectively) out of 144 papers. The papers that were not written in English (except for non-English articles with dose tables in English) or did not include the dose to bone marrow, brain, salivary glands, and thyroid and effective doses were excluded from the review process. After the exclusion, we finally used 3, 9, and 11 papers providing organ and effective doses for intraoral, panoramic, and CBCT procedures, respectively.

2.2. Data Collection. The following data were extracted from the papers:

(i) Dosimetry methods: simulation or measurement

(ii) Imaging machine model

(iii) Age represented by physical (measurement) or computational (simulation) phantoms: we denoted the age of 35 as the minimum age for all adult phantoms, which is the International Commission on Radiological Protection (ICRP) reference age of adults [6]

(iv) Dose calculation program for simulation studies or dosimeter type for measurement studies

(v) Beam rotation angle (only for CBCT)

(vi) Imaging protocol

(vii) Dose area product (DAP) $\left(\mathrm{mGy}-\mathrm{cm}^{2}\right)$ (for intraoral and CBCT)

(viii) Tube potential $(\mathrm{kVp})$

(ix) The width and height of field of view (FOV) $(\mathrm{cm})$ (for intraoral and CBCT)

(x) Effective dose $(\mathrm{E})(\mu \mathrm{Sv})$

(xi) Doses to the bone marrow, brain, salivary glands, and thyroid $(\mu \mathrm{Gy})$

When a single paper provided multiple dose data in multiple categories, the dose in each category was considered a separate dose set. Simulation and measurement data were analyzed separately when both were reported in a single paper. When an effective dose was missing but organ doses were reported, an effective dose was derived from the organ doses using tissue weighting factors from ICRP Publication 103 [4]: 0.12 (bone marrow), 0.01 (brain), 0.01 (salivary glands), and 0.04 (thyroid). We assumed zero doses for other organs outside the head region in the calculation of the effective dose. The extracted data were tabulated in three detailed tables for intraoral, panoramic, and CBCT, respectively.

To efficiently analyze the doses, we averaged the organ and effective doses over different data sources. As for CBCT, which had more available data for different phantom ages and FOVthan intraoral and panoramic, we further arranged organ and effective doses by phantom age group (children and adults) and/or the area (height $x$ width) of FOV (small $<50 \mathrm{~cm}^{2}$, medium $50-150 \mathrm{~cm}^{2}$, and large $>150 \mathrm{~cm}^{2}$ ).

2.3. Monte Carlo Simulation of Dental Imaging Procedures. We conducted Monte Carlo simulations of intraoral, panoramic, and CBCT imaging procedures by using a 
computational human head phantom and multimodal imaging-based detailed anatomical (MIDA) [7]combined with a Monte Carlo radiation transport code, MC-GPU [8]. The voxel resolution of the head phantom was $0.5 \times 0.5 \times 0.5 \mathrm{~mm}^{3}$. Key technical parameters for Monte Carlo simulations that were collected from literature are summarized in Table 1. In the case of panoramic imaging, a simplified image acquisition was modeled by concatenating 9,153 simulations with a 1-pixel-wide field of view of $10 \times 0.05 \mathrm{~cm}^{2}$ instead of the realistic field of view of $10 \times 0.2 \mathrm{~cm}^{2}$ since the image overlap in real machines could not be reliably simulated. Our simulations had two purposes: to evaluate the proportion of the dose distribution among different tissues during the three imaging modalities and to visualize dose distribution across the head anatomy in the web program.

2.4. Development of a Web-Based Dose Calculator. After the summary dose tables were established, we developed a webbased dose calculation program to allow for convenient access to the organ and effective doses and comparison of the dental doses with doses from other radiation sources.

The web program was designed to allow an input from the user for the following parameters: type of imaging modalities, number of image sets, patient age group (child or adult), and size of imaging region, which is the area of the FOV (small $<50 \mathrm{~cm}^{2}$, medium $50-150 \mathrm{~cm}^{2}$, and large $>150 \mathrm{~cm}^{2}$ ). The last two parameters were only used for CBCT dose as the dose data for intraoral and panoramic imaging were not enough to be stratified by age and FOV. The user has the option of "I do not know" for the patient age group and FOV, in which case the average dose of the age groups and/or the size of FOV were presented.

Based on the input data from a user, the web application presents the following information:

(i) Dose delivered to the bone marrow, brain, salivary glands, and thyroid and effective dose; the doses are calculated by multiplying the dose per imaging by the number of image sets inputted by a user. Limited pediatric data points were available for intraoral and panoramic, andonly a total of 22 pediatric data points were extracted for CBCT. Since the data points were not enough to derive age dependency of dose for finer age resolution, we combined the 22 data points for $\mathrm{CBCT}$ into the pediatric age group. In the case of CBCT, when the user selects both age group and FOV, age and FOV dependent doses are displayed. FOV-averaged doses are displayed when a user selects "I do not know" for FOV. Age-averaged dose is displayed when a user selects "I do not know" for age.

(ii) $2 \mathrm{D}$ and $3 \mathrm{D}$ dose distribution for the selected imaging procedures and the fraction of dose delivered to different tissues

(iii) Comparison of the total effective dose (effective dose multiplied by the number of image sets) with that from other radiation sources: $37 \mu \mathrm{Sv}$ (Londonto-New York flight), $100 \mu \mathrm{Sv}$ (chest X-ray) and $3110 \mu \mathrm{Sv}$ (annual natural background) [9]

The web dose calculator was developed using the commercial cross-platform language, Xojo (Xojo, Inc., Austin, TX). The Xojo development tool provides a graphical user interface-based programming environment to develop multiplatform apps for macOS, Windows, Linux, and Web. We used the web application platform to develop our web-based dose calculator. We created two versions of the web interface for web browsers on a personal computer and a smart phone to account for differences in screen size. The web application was deployed through Xojo Cloud hosting, which was connected to the domain name http://dentaldose.org. Figure 1 shows the workflow of the web program, where the user input data and output data are described.

\section{Results}

We tabulated technical parameters and doses for a total of 4 , 18 , and 51 dose sets for intraoral (Table 2), panoramic (Table 3), and CBCT (Table 4) procedures, respectively.

3.1. Technical Parameters and Methods Used in Dosimetry Studies. About 56\% $(n=57)$ of the dose sets were from measurements, and the remaining $44 \%(n=44)$ were from simulation studies. In the measurement studies, different types of dosimeters were used: the thermoluminescent dosimeter (TLD) (86\%), the optically stimulated luminescent dosimeter (OSLD) (7\%), the Gafchromic film (5\%), and the metal-oxide-silicon field-effect transistor (MOSFET) (2\%). The physical head phantoms used for measurements included the Alderson Radiation Therapy (ART) phantom (Radiology Support Devices Inc., Long Beach, CA) (70\%), the ATOM adult and child phantoms (CIRS, Norfolk, VA) (20\%), and CDP-R1 (Chengdu Fangtuo Simulation Technology Company Limited, China) (10\%). In the simulation studies, the EGS program [27] produced about $60 \%$ of all dose sets followed by MCNP [28] (22\%) and PCXMC [29] (STUK, Helsinki, Finland) (18\%). A variety of computational head phantoms were used for the simulation studies: inhouse head phantoms developed from patient CT images (44\%), the Zubal head phantom [30] (22\%), the Oak Ridge National Laboratory (ORNL)-stylized phantoms (18\%), and the ICRP adult phantoms [31] (16\%). The tube potential for intraoral imaging ranged from 60 to $70 \mathrm{kVp}$. The panoramic and $\mathrm{CBCT}$ scans used $62-73 \mathrm{kVp}$ and $70-120 \mathrm{kVp}$, respectively. In the case of CBCT, the width and height of the FOV ranged from about 4 to $26 \mathrm{~cm}$ and the area (width $\times$ height) ranged from about $15[11]$ to $600[24] \mathrm{cm}^{2}$. The beam rotation angle for CBCT was between $180^{\circ}$ and $360^{\circ}$. The DAP for CBCT ranged from 91 to $1080 \mathrm{mGy}-\mathrm{cm}^{2}$.

3.2. Organ and Effective Doses. The organ and effective doses reported in the literature are summarized in Table 5. Mean effective doses delivered by intraoral, $1.32(0.60-2.56) \mu \mathrm{Sv}$, 
TABLE 1: Technical parameters for intraoral, panoramic, and cone-beam computed tomography collected from literature, which were used for Monte Carlo simulations conducted using MC-GPU to estimate dose distribution within the head.

\begin{tabular}{lccc}
\hline Parameters & Intraoral & Panoramic & Cone-beam computed tomography \\
\hline X-ray energy (kVp) & 60 & 73 & 90 \\
Filtration (mm Al) & 3.5 & 2.5 & 2.8 \\
FOV $\left(\mathrm{cm}^{2}\right)$ & $4 \times 3$ & $10 \times 0.2$ & $10 \times 10$ \\
SRD $^{1}(\mathrm{~cm})$ & 75 & 35 & 50 \\
Rotation angle per view (degree) & $0^{2}$ & 240 & 360 \\
Number of views per acquisition & 1 & 9153 & 360 \\
Number of X-rays per simulated view & $10^{11}$ & $5 \times 10^{7}$ & $5 \times 10^{9}$ \\
Total simulation time (min) & 33 & 143 & 760 \\
\hline
\end{tabular}

${ }^{1}$ Source-to-rotation axis distance. ${ }^{2}$ The rectangular field was rotated $30^{\circ}$ cranially. ${ }^{3}$ Simulation run in an NVIDIA GeForce GTX 1080 GPU.

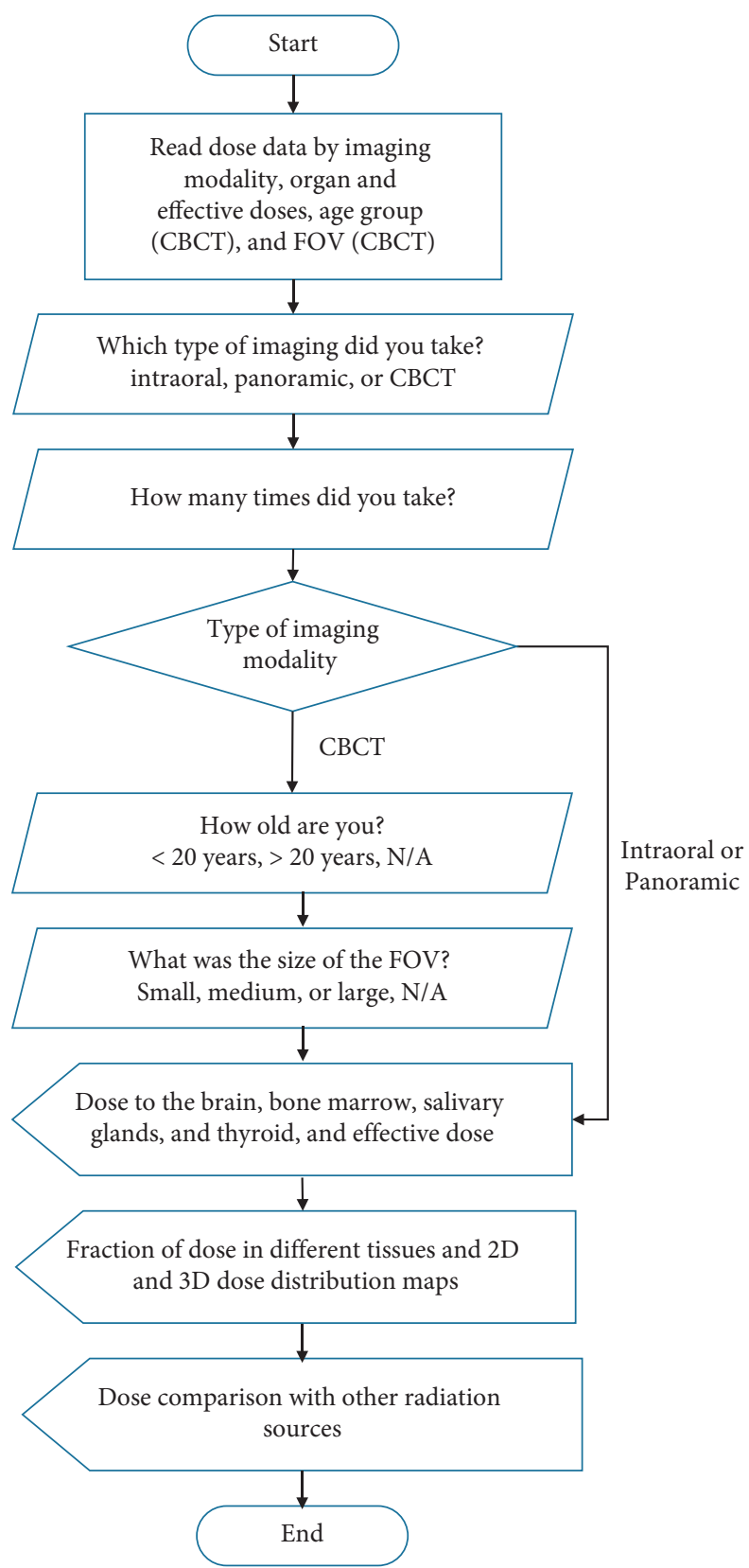

FIgURE 1: Flowchart of the web application for dental radiation dose calculations and dose display. 


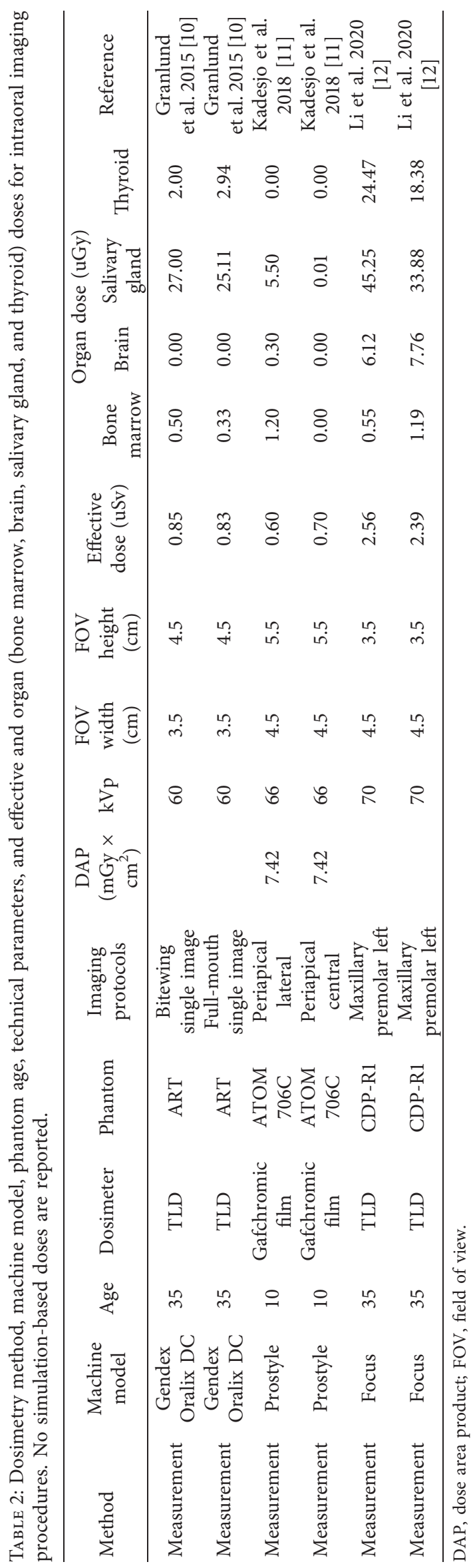




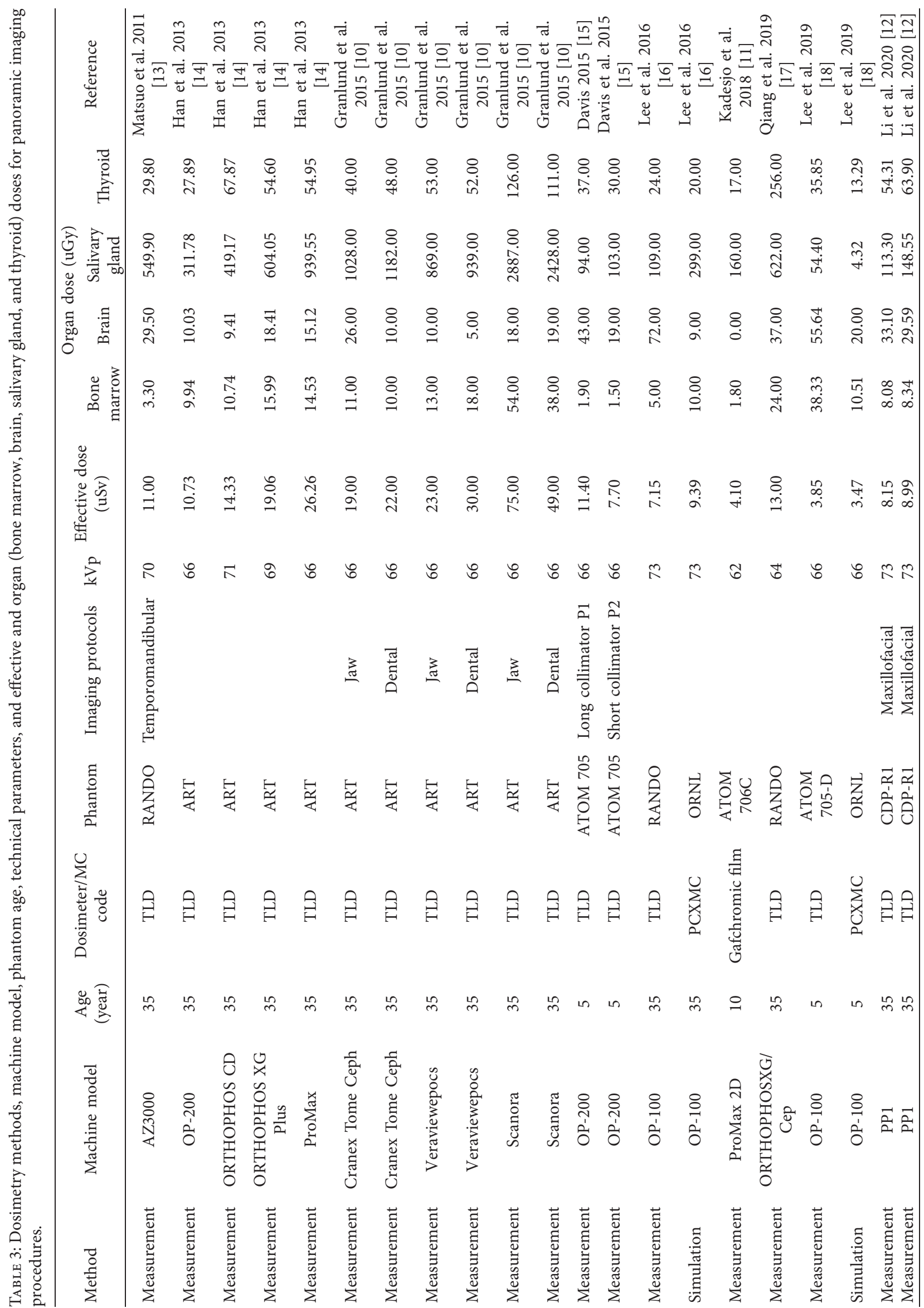




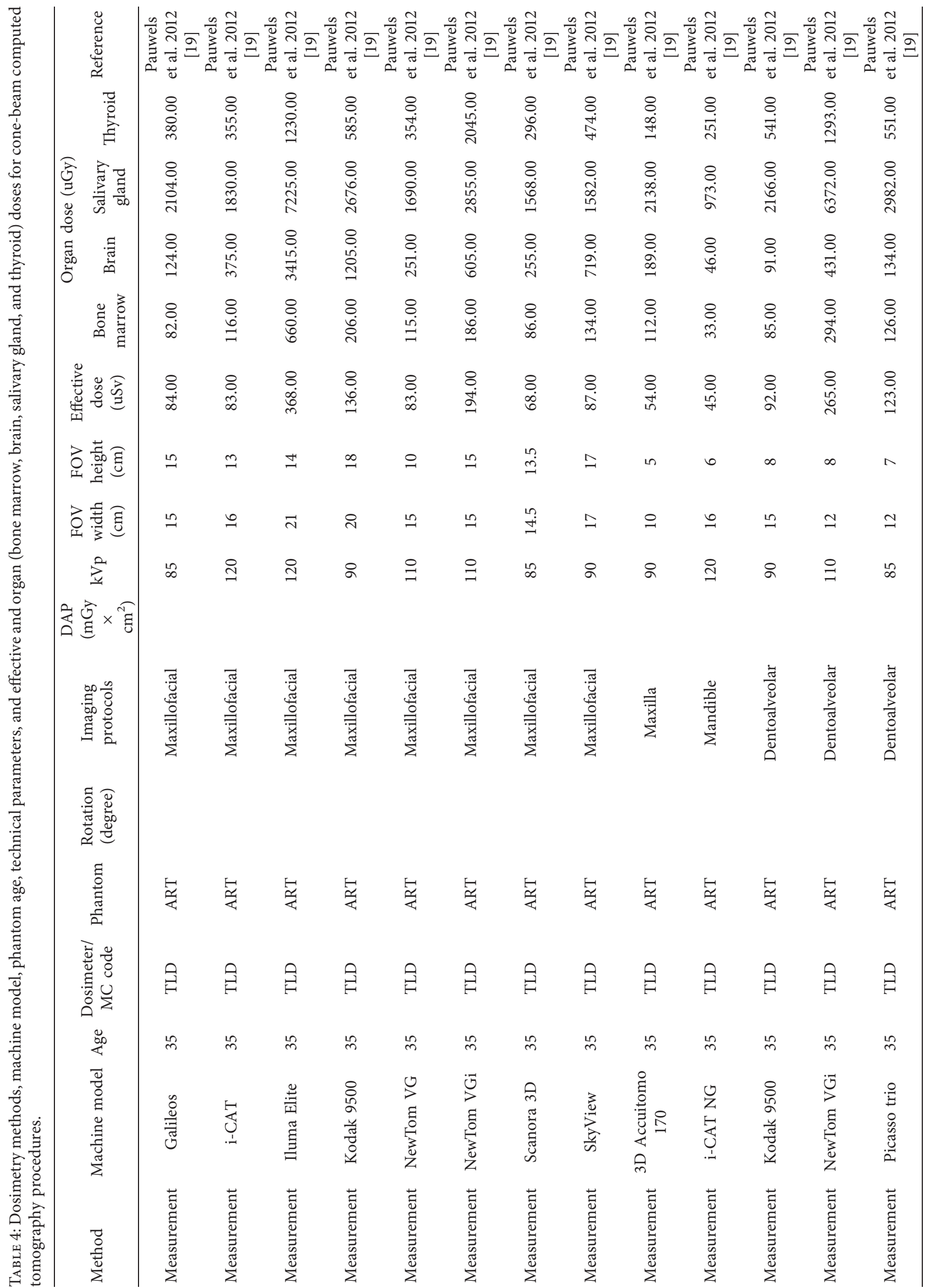




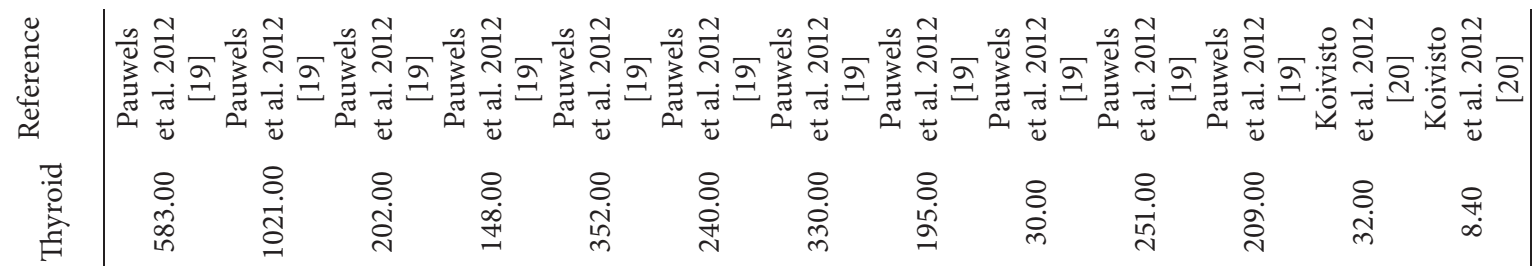

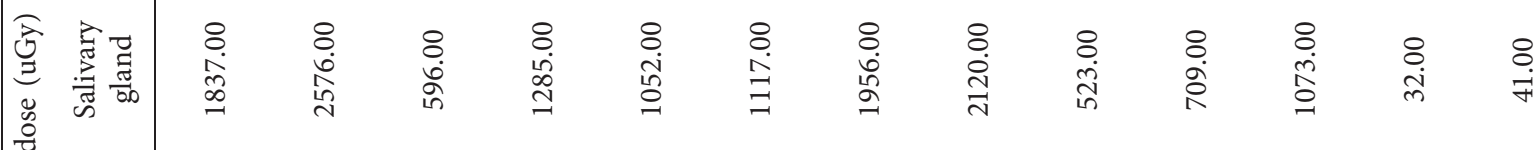

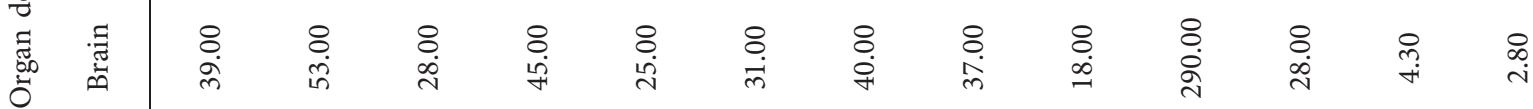

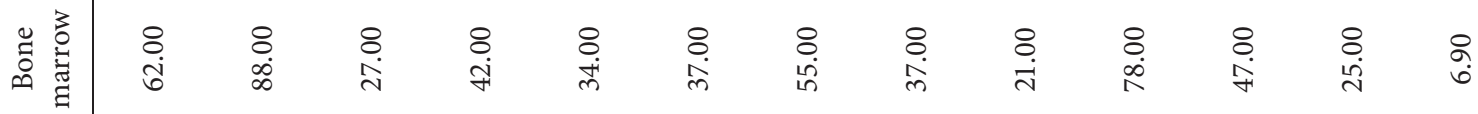

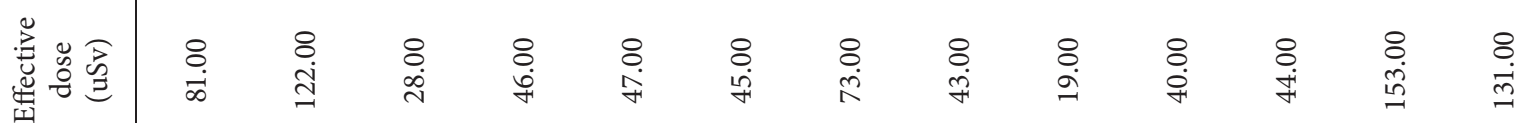

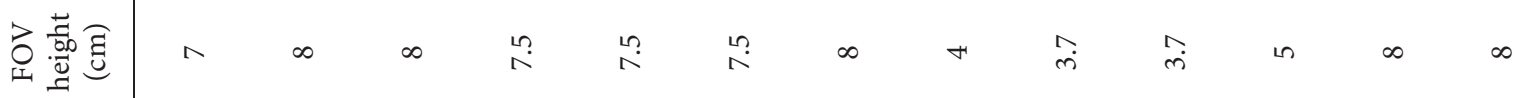

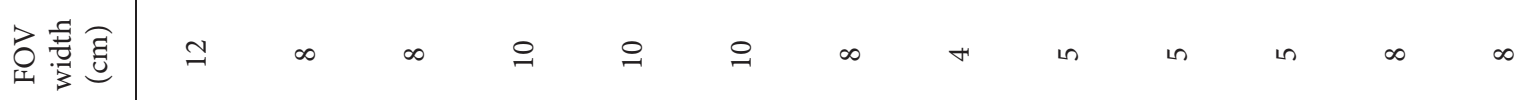

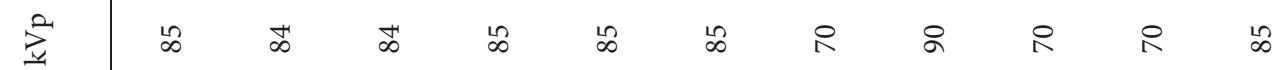

运馬 $\times$ 苞

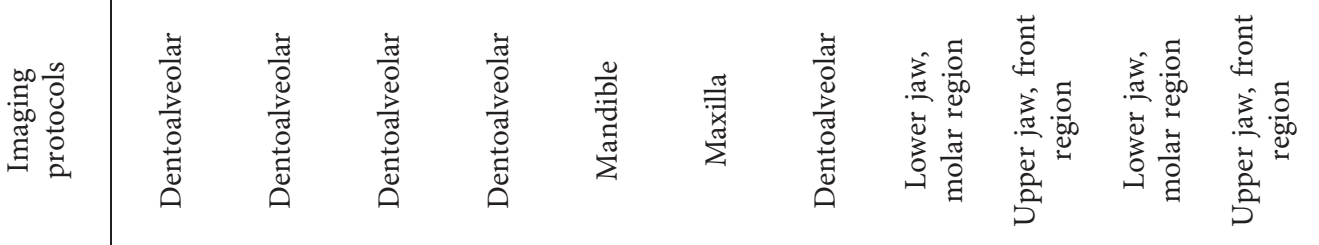

$\stackrel{\Xi}{\stackrel{\Xi}{\rightleftarrows}}$

莺 莺

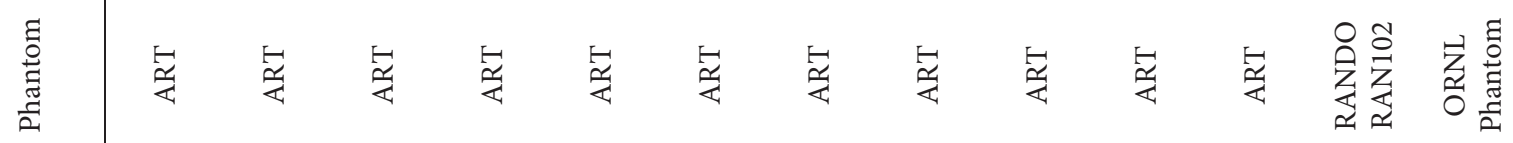

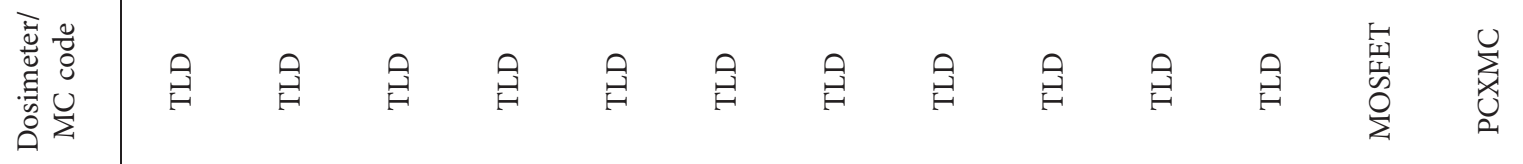

要

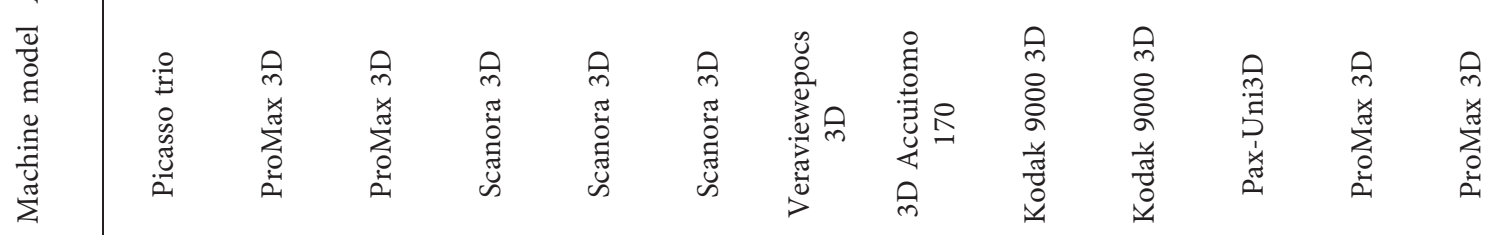

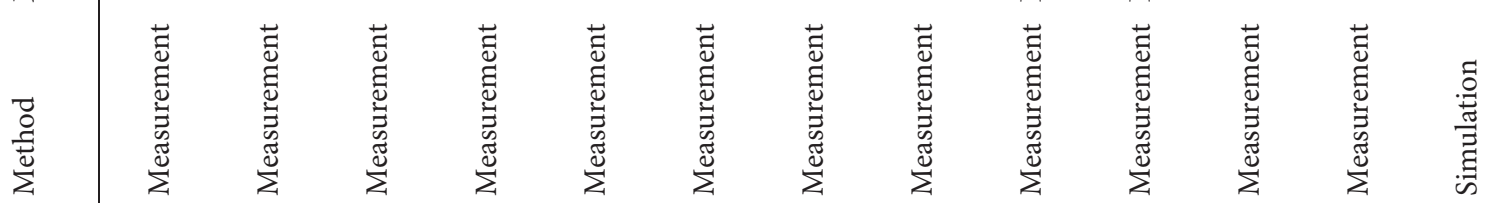




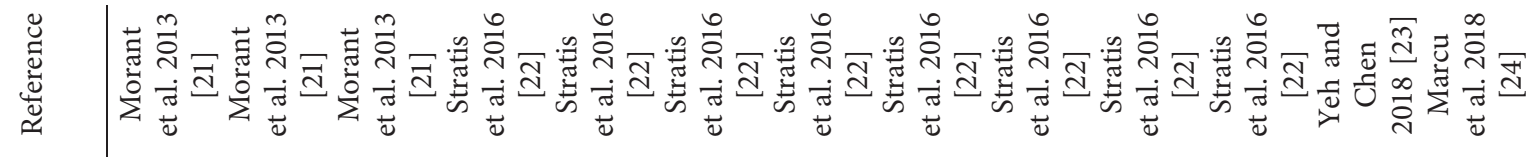

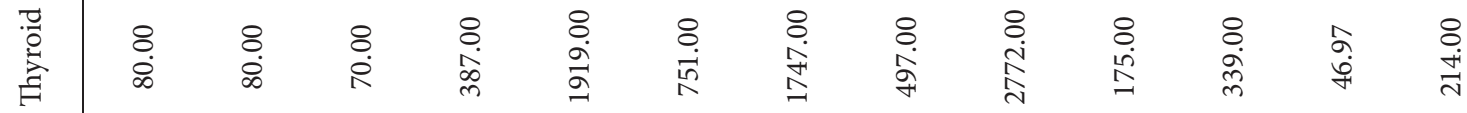

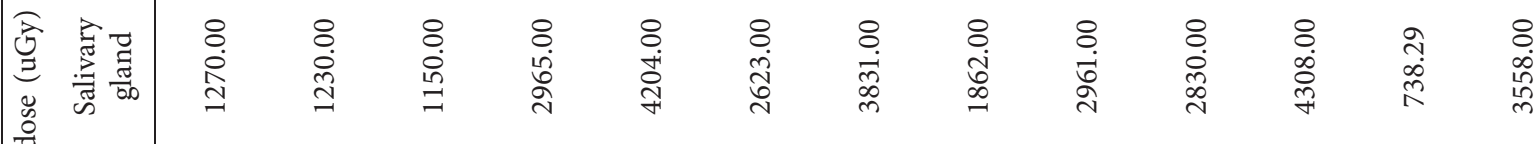

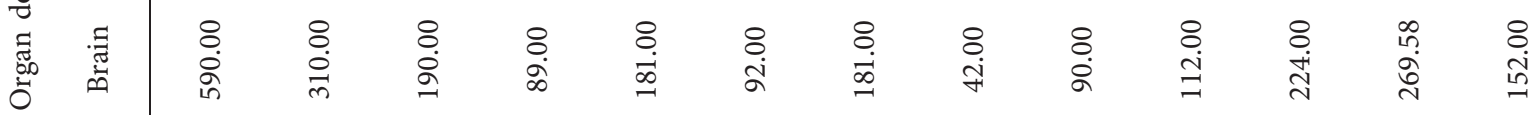

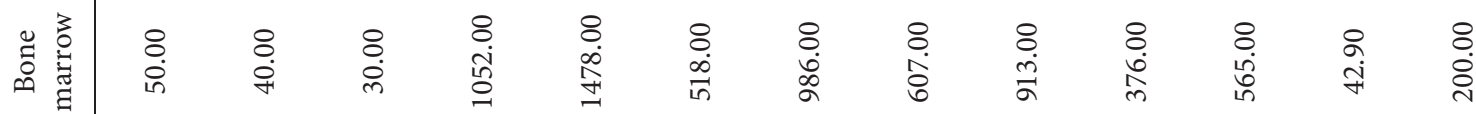

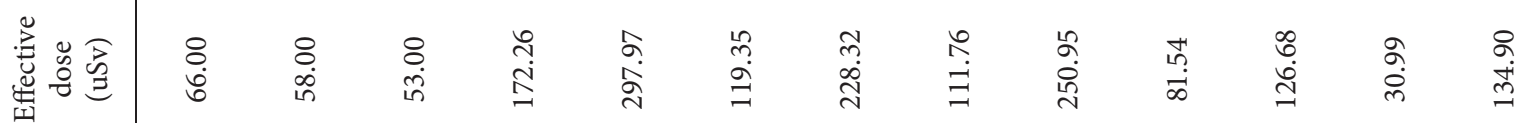

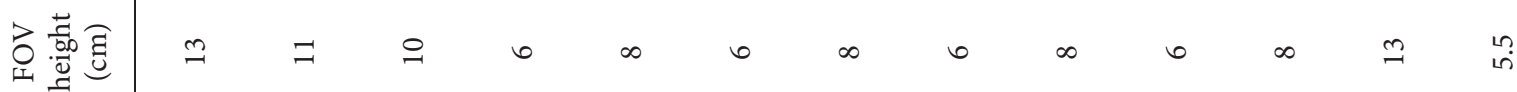
完毒泀 in

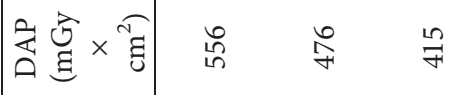
ஃ ஃ ஃ ஃ ஃ ஃ ஃ ஃ ஃ

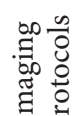

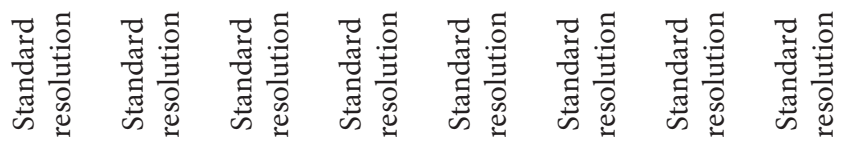
空
范
芯

ㅇํㅇ

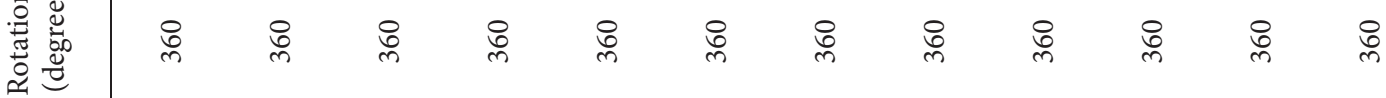

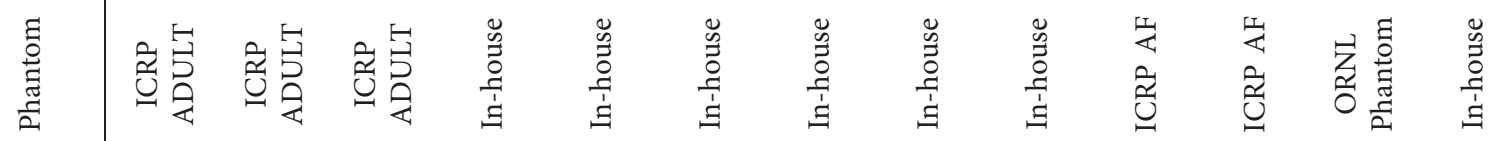

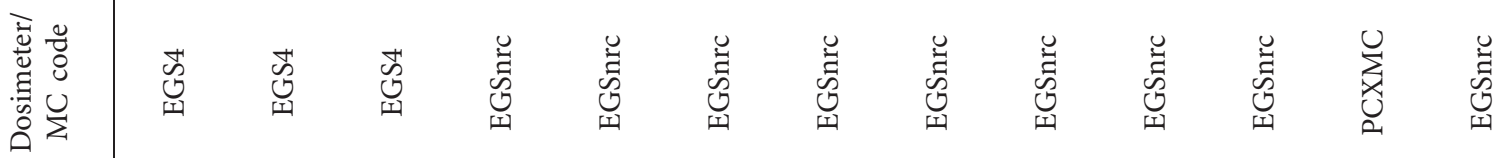

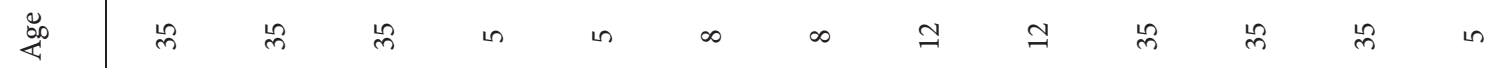

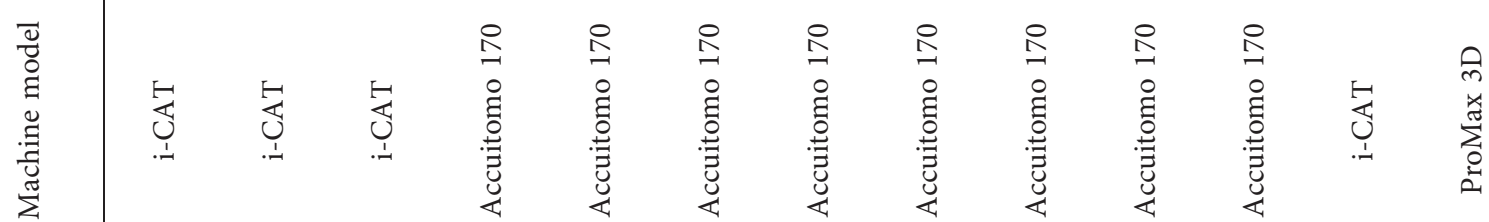

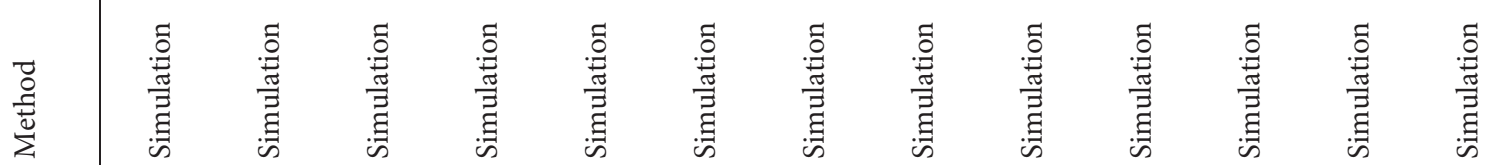




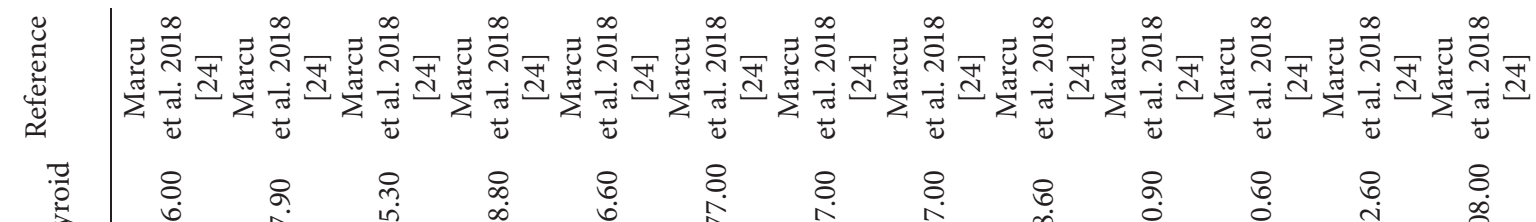

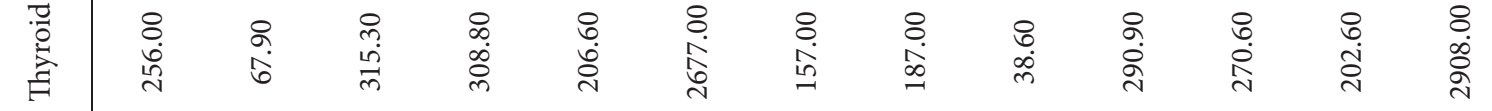

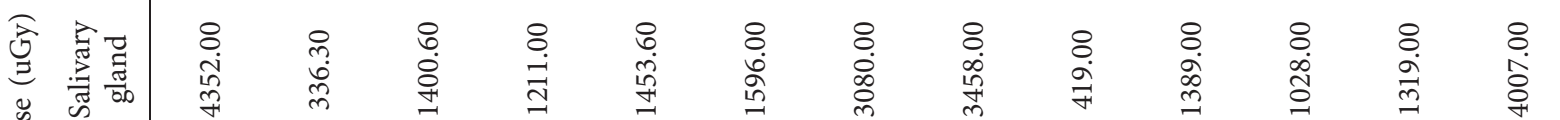

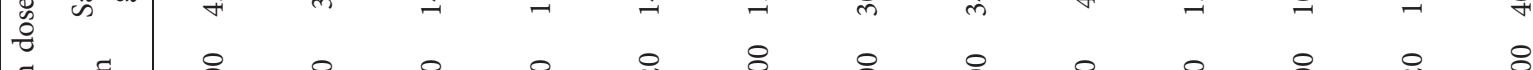

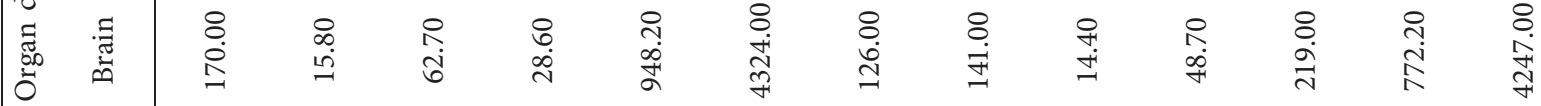

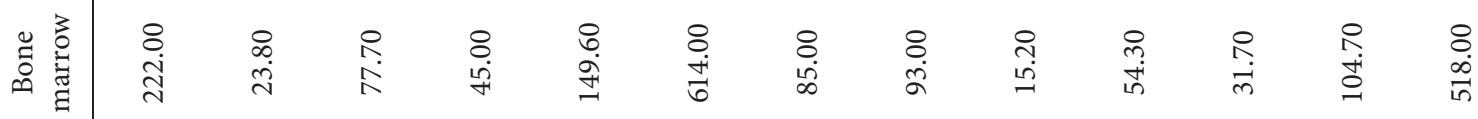

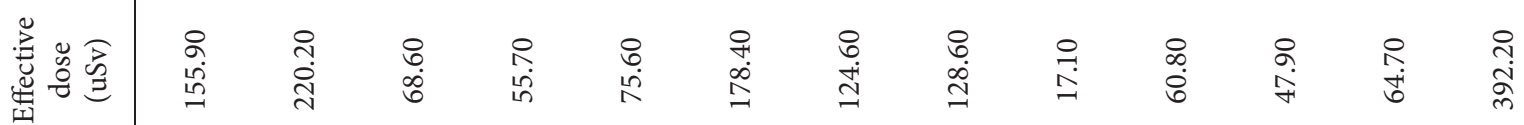

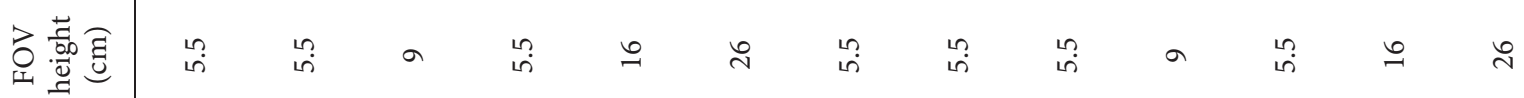

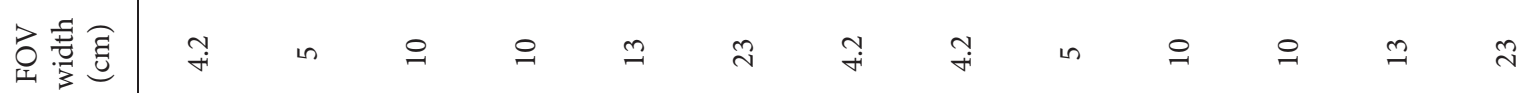
咅 它氮 $\times$ 胥

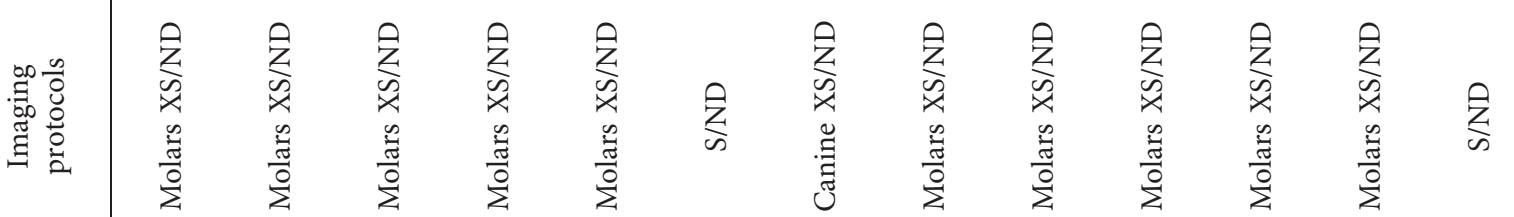

(ี) 跑 密

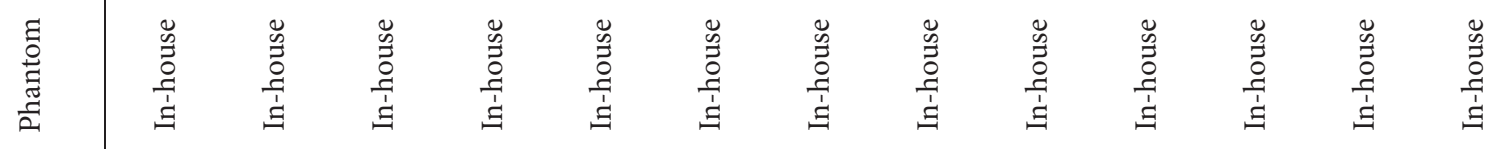

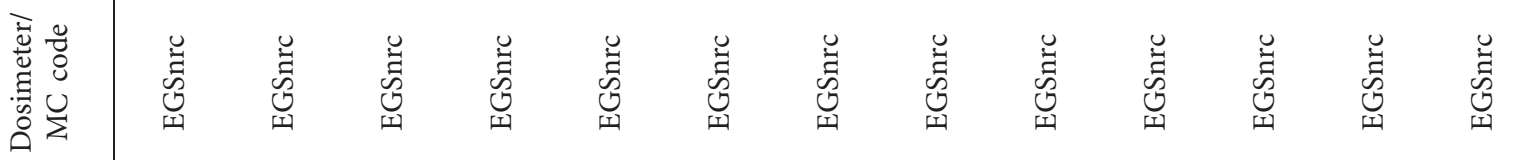

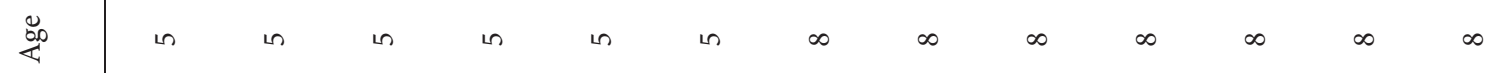
छँ

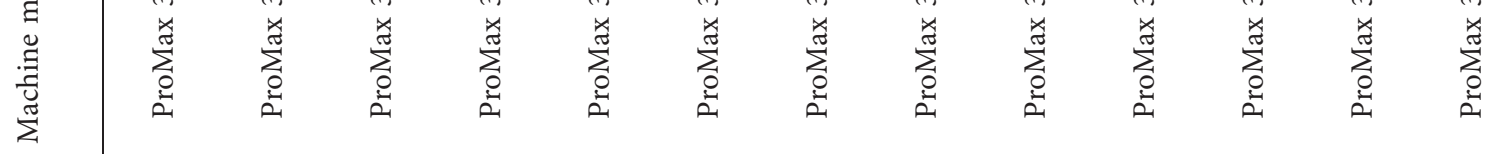

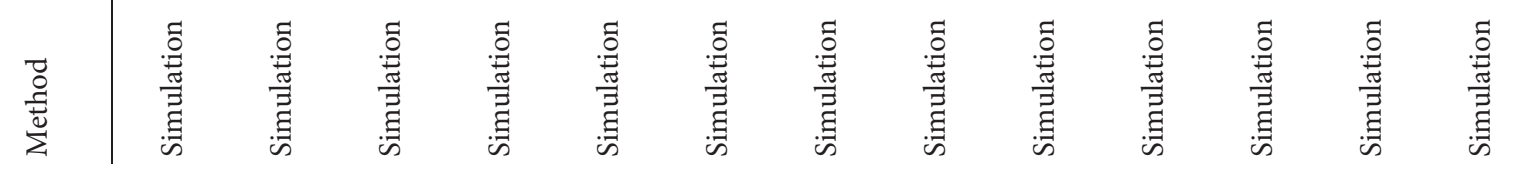




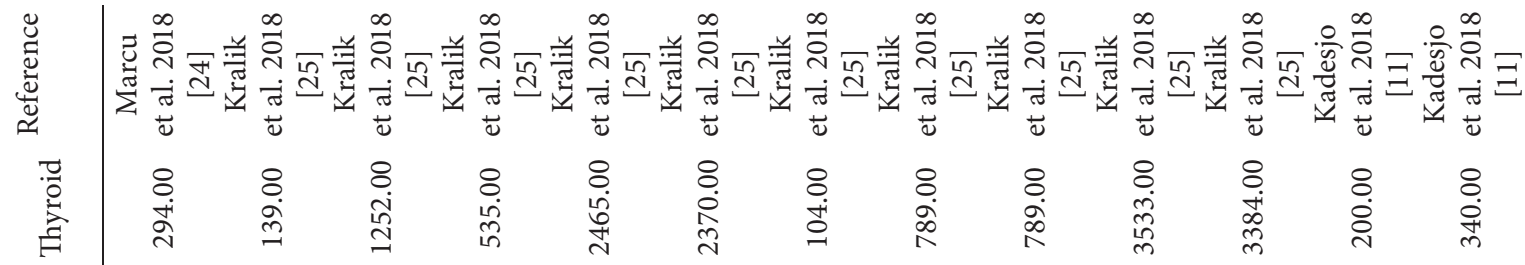

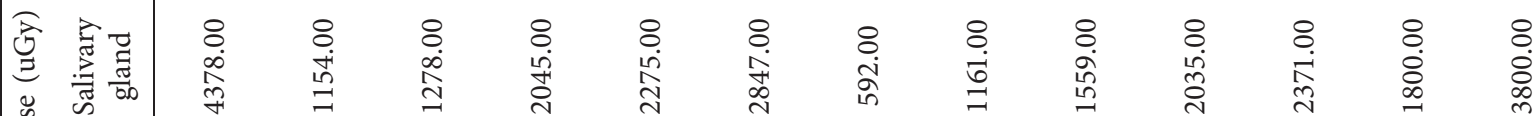

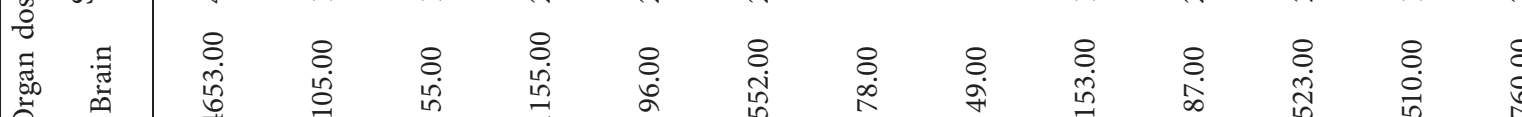

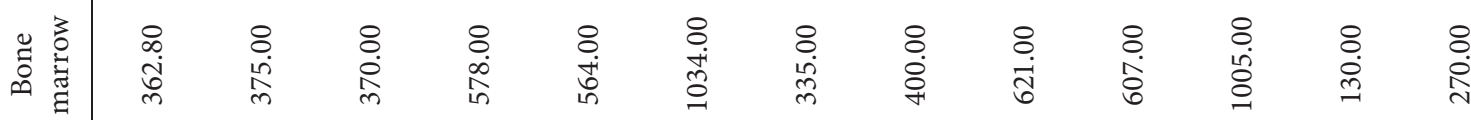

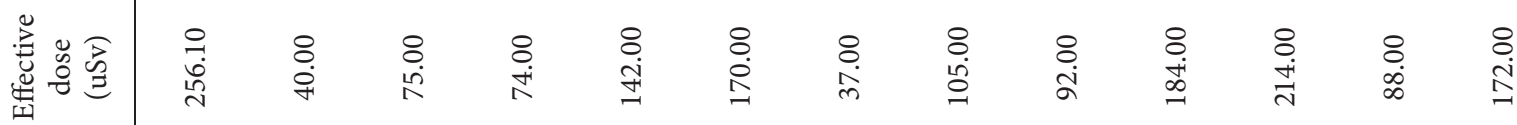

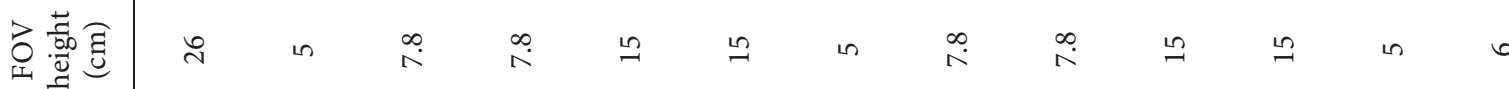

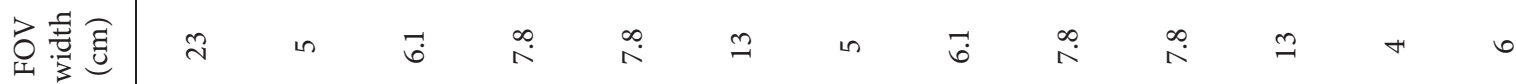
咅

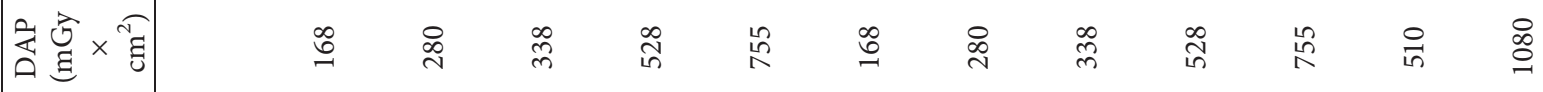

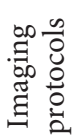

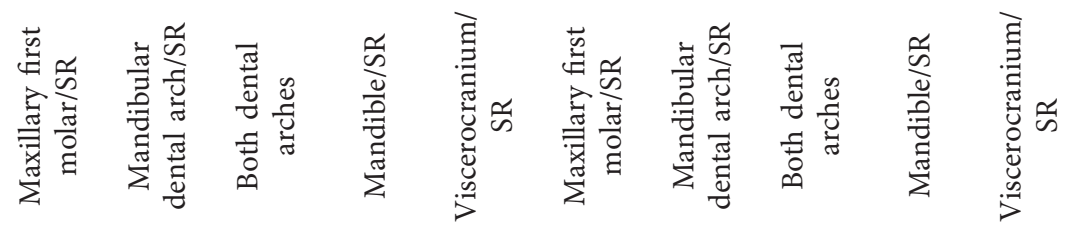

.

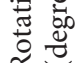

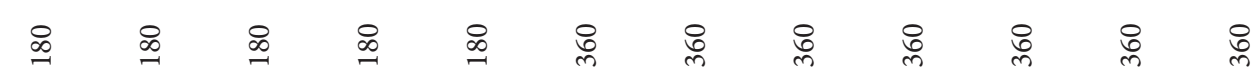

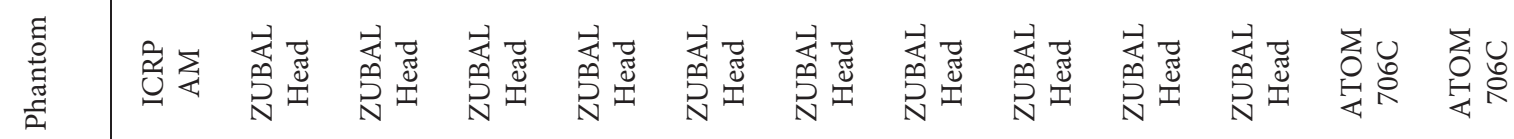

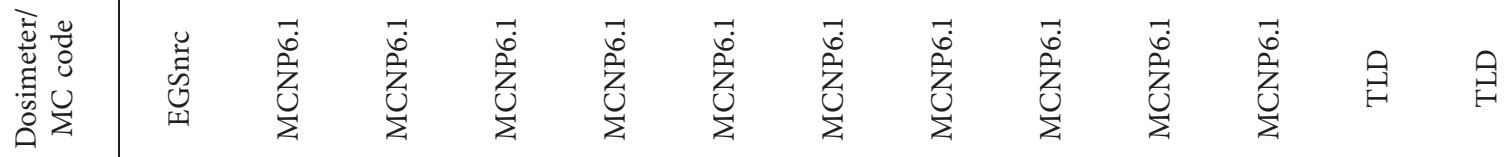

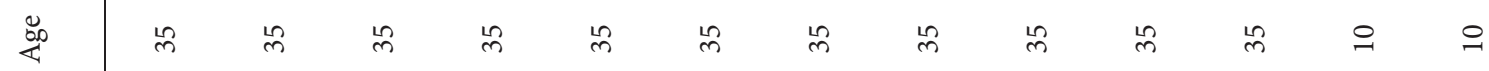

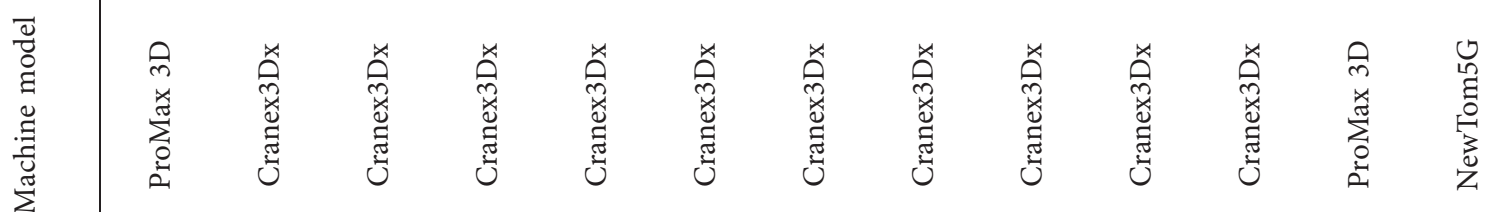

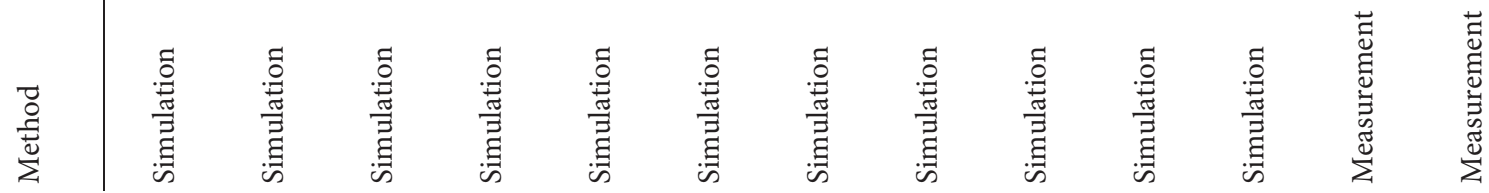




\begin{tabular}{|c|c|c|c|c|c|c|c|c|c|c|c|c|}
\hline & \multicolumn{12}{|c|}{ 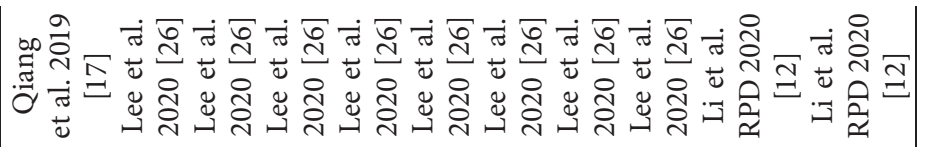 } \\
\hline & 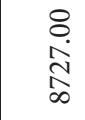 & $\begin{array}{l}8 \\
8 \\
8 \\
4\end{array}$ & $\begin{array}{l}8 \\
\dot{\infty} \\
i\end{array}$ & $\begin{array}{l}8 \\
\stackrel{\pi}{\pi}\end{array}$ & $\begin{array}{l}8 \\
\stackrel{0}{7} \\
7\end{array}$ & 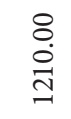 & $\begin{array}{l}8 \\
\stackrel{0}{0} \\
\infty \\
10\end{array}$ & $\begin{array}{l}8 \\
0 \\
0 \\
0 \\
0\end{array}$ & $\begin{array}{l}8 \\
8 \\
8 \\
0\end{array}$ & \begin{tabular}{l}
5 \\
\multirow{0}{0}{} \\
$\stackrel{+}{+}$
\end{tabular} & \begin{tabular}{l}
$\infty$ \\
$b$ \\
$i$ \\
$i$ \\
\multirow{f}{f}{}
\end{tabular} & \\
\hline 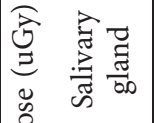 & $\begin{array}{l}8 \\
\stackrel{8}{1} \\
\\
\end{array}$ & $\begin{array}{l}8 \\
8 \\
0 \\
19 \\
4\end{array}$ & $\begin{array}{l}8 \\
8 \\
\stackrel{0}{2} \\
2\end{array}$ & $\begin{array}{l}8 \\
0 \\
0 \\
i n \\
i n\end{array}$ & 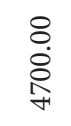 & $\begin{array}{l}8 \\
\dot{0} \\
\dot{m}\end{array}$ & $\begin{array}{l}8 \\
\vdots \\
\vdots \\
2\end{array}$ & $\begin{array}{l}8 \\
8 \\
\stackrel{4}{1} \\
\text { अ }\end{array}$ & $\begin{array}{l}8 \\
\dot{m} \\
\text { ळ. }\end{array}$ & $\stackrel{\stackrel{N}{N}}{\stackrel{\Xi}{=}}$ & $\begin{array}{l}\stackrel{\mathscr{L}}{\infty} \\
\stackrel{+}{+} \\
\stackrel{m}{-}\end{array}$ & \\
\hline 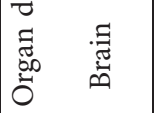 & 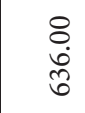 & $\begin{array}{l}8 \\
8 \\
8 \\
8 \\
+1\end{array}$ & $\begin{array}{l}\stackrel{0}{0} \\
\stackrel{\oplus}{\sim}\end{array}$ & 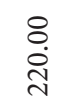 & $\begin{array}{l}8 \\
\stackrel{0}{0} \\
\text { i }\end{array}$ & $\begin{array}{l}8 \\
\stackrel{0}{0} \\
0 \\
0\end{array}$ & $\begin{array}{l}8 \\
\stackrel{0}{0} \\
\text { त }\end{array}$ & $\begin{array}{l}8 \\
\stackrel{\infty}{\infty} \\
\infty\end{array}$ & $\underset{\substack{8 \\
\text { in }}}{8}$ & 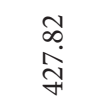 & 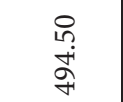 & \\
\hline 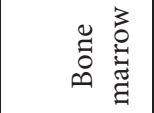 & 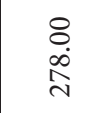 & $\begin{array}{l}8 \\
\stackrel{0}{0} \\
\infty\end{array}$ & $\stackrel{8}{\circ}$ & $\begin{array}{l}8 \\
\stackrel{\leftrightarrow}{0}\end{array}$ & $\stackrel{8}{\circ}$ & $\stackrel{8}{\circ}$ & $\stackrel{8}{\circ}$ & $\stackrel{8}{\circ}$ & $\stackrel{8}{\circ}$ & $\begin{array}{l}\text { กิ } \\
\text { فำ }\end{array}$ & $\stackrel{\substack{0 \\
=}}{=}$ & \\
\hline 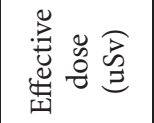 & $\begin{array}{l}8 \\
\text { ஸें } \\
\text { ஸे }\end{array}$ & $\begin{array}{l}\text { ̊. } \\
\stackrel{0}{0}\end{array}$ & $\begin{array}{l}\text { ơ } \\
\text { +i }\end{array}$ & $\begin{array}{l}8 \\
\infty \\
\infty \\
\stackrel{0}{-}\end{array}$ & 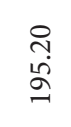 & 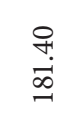 & $\begin{array}{l}\stackrel{R}{8} \\
\stackrel{8}{8}\end{array}$ & $\begin{array}{l}\text { in } \\
\text { in } \\
\text { ते }\end{array}$ & $\begin{array}{l}\infty \\
\stackrel{\infty}{\vec{v}} \\
\stackrel{\sim}{v}\end{array}$ & $\begin{array}{l}\text { ro } \\
\dot{0} \\
i\end{array}$ & $\stackrel{\infty}{\omega^{\circ}}$ & \\
\hline 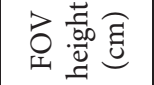 & $\stackrel{10}{-1}$ & $\stackrel{n}{n}$ & 으 & $\circ$ & $ㅇ$ & $\stackrel{n}{m}$ & 으 & 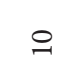 & $\stackrel{\circ}{\circ}$ & $\begin{array}{l}n \\
\infty \\
\infty\end{array}$ & 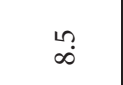 & \\
\hline 它吾氞 & $\stackrel{10}{-1}$ & $\triangleq$ & 으 & $\stackrel{\circ}{-}$ & $ㅇ$ & $\bumpeq$ & $\stackrel{\circ}{\circ}$ & $\stackrel{\bullet}{-}$ & 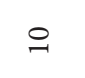 & $\stackrel{n}{ \pm}$ & $\stackrel{n}{\underset{+}{*}}$ & \\
\hline$\sum^{2}$ & $\infty$ & \& & \& & $\infty$ & $\infty$ & \& & \& & $\infty$ & $\infty$ & త్తి & తి & \\
\hline 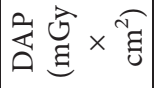 & & $\stackrel{\vec{n}}{\mathrm{~N}}$ & $\vec{\sigma}$ & 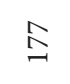 & $\sigma$ & $\stackrel{\sqrt[n]{n}}{ }$ & $\sigma$ & 총 & $\sigma$ & & & \\
\hline 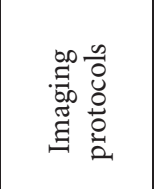 & 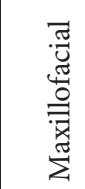 & 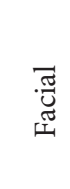 & 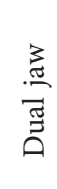 & 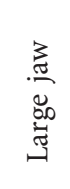 & 胥 & 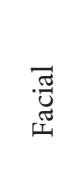 & 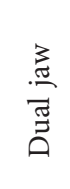 & 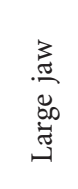 & 层 & 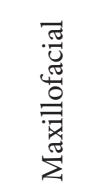 & 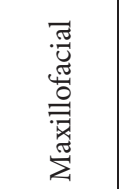 & \\
\hline 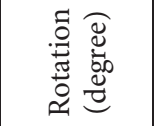 & & 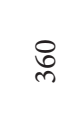 & d & : & 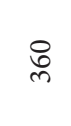 & 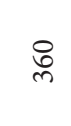 & $\stackrel{8}{\stackrel{0}{0}}$ & : & i & & & \\
\hline 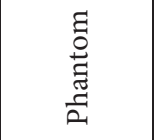 & $\stackrel{\bigcirc}{\gtrless}$ & 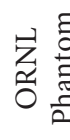 & & & & & & $\sum_{0}$ & 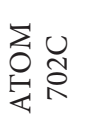 & 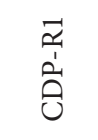 & 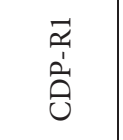 & \\
\hline 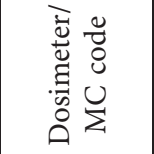 & 肖 & $\sum_{\substack{x \\
\hdashline}}^{u}$ & 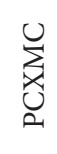 & $\sum_{\substack{ג \\
\hdashline}}^{u}$ & $\sum_{\substack{x \\
0}}^{u}$ & 今ે & คิ & 䓢 & 今ે & 帠 & 宣 & \\
\hline 范 & $\stackrel{n}{m}$ & $\stackrel{m}{m}$ & $\stackrel{n}{m}$ & $\stackrel{m}{m}$ & $\stackrel{m}{m}$ & $\ddot{n}$ & $\stackrel{m}{m}$ & $\stackrel{m}{n}$ & $\stackrel{m}{m}$ & $\stackrel{m}{m}$ & $\stackrel{m}{m}$ & \\
\hline 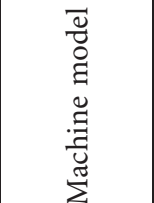 & 总 & $\begin{array}{l}\text { ठे } \\
\text { oे }\end{array}$ & $\begin{array}{l}8 \\
\text { ò } \\
\text { రे }\end{array}$ & 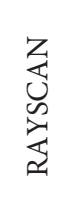 & 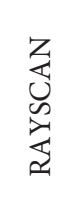 & $\begin{array}{l}\text { \&े } \\
\text { òे }\end{array}$ & $\begin{array}{l}8 \\
\text { ò } \\
\text { லे }\end{array}$ & 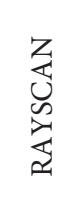 & 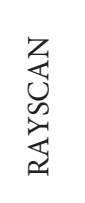 & 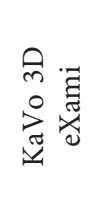 & 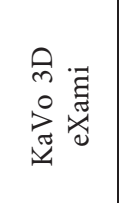 & \\
\hline $\begin{array}{l}\overline{0} \\
\text { 节 } \\
\sum^{2}\end{array}$ & 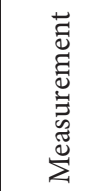 & 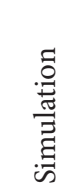 & 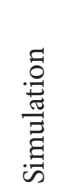 & 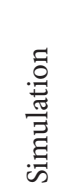 & 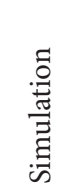 & 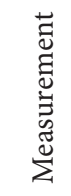 & 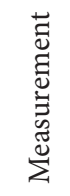 & 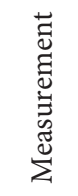 & 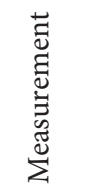 & 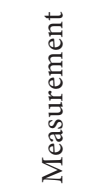 & 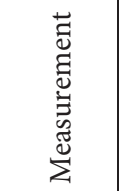 & \\
\hline
\end{tabular}


TABle 5: Minimum, mean, and maximum values of effective and organ (bone marrow, brain, salivary gland, and thyroid) doses from intraoral, panoramic, and cone-beam computed tomography procedures reported in the selected publications.

\begin{tabular}{lcccccc}
\hline & & \multirow{2}{*}{ Procedures } & & & \multicolumn{3}{c}{ Organ dose $(\mu \mathrm{Gy})$} \\
& & Effective dose $(\mu \mathrm{Sv})$ & Bone marrow & Brain & Salivary gland & Thyroid \\
\hline \multirow{3}{*}{ Intraoral $(n=6)$} & Mean & 1.32 & 0.63 & 2.36 & 22.79 & 7.97 \\
& Min & 0.60 & 0.00 & 0.00 & 0.01 & 0.00 \\
& Max & 2.56 & 1.20 & 7.76 & 45.25 & 24.47 \\
\hline \multirow{3}{*}{ Panoramic $(n=21)$} & Mean & 17.93 & 14.66 & 23.28 & 660.24 & 57.93 \\
& Min & 3.47 & 1.50 & 0.00 & 4.32 & 13.29 \\
& Max & 75.00 & 54.00 & 72.00 & 2887.00 & 256.00 \\
\hline \multirow{3}{*}{ Cone-beam computed tomography $(n=76)$} & Mean & 121.09 & 254.78 & 471.65 & 2333.95 & 811.16 \\
& Min & 17.10 & 6.90 & 2.80 & 32.00 & 8.40 \\
& Max & 392.20 & 1478.00 & 4653.00 & 7775.00 & 8727.00 \\
\hline
\end{tabular}

and panoramic, $17.93(3.47-75.00) \mu \mathrm{Sv}$, procedures are about $1 \%$ and $15 \%$ of that delivered by CBCT, 121.09 (17.10-392.20) $\mu \mathrm{Sv}$. Among the three imaging modalities, the salivary glands received the greatest dose: $22.79 \mu \mathrm{Gy}$ (intraoral), $660.24 \mu \mathrm{Gy}$ (panoramic), and $2333.95 \mu \mathrm{Gy}$ (CBCT). Among the four organs of interest, the smallest dose was delivered to the bone marrow, except for intraoral where the brain received the smallest dose.

In CBCT imaging, the child phantoms tended to receive greater doses compared with the adult phantoms, except for the salivary glands and thyroid doses (Table 6). The child phantoms received about $29 \%$ greater effective dose than the adult phantoms. The bone marrow dose of the child phantoms was about $80 \%$ greater than that of the adult phantom.

The effective dose for the larger FOV in CBCT is greater than that for the smaller FOV (Table 7). The effective dose for the large FOV (greater than $150 \mathrm{~cm}^{2}$ ) is about 1.6 times greater than that for the small FOV (less than $50 \mathrm{~cm}^{2}$ ). The brain dose for the large FOV is about eight times greater than that for the small FOV.

Table 8 shows the age- and FOV size-dependent organ and effective doses. A similar trend by age group shown in Table 6 (child's dose is greater than adult's dose) and by FOV size shown in Table 7 (large FOV gives greater dose than small FOV) is also observed.

3.3. Monte Carlo Dose Distribution. 2D dose distribution at the level of the center of the lower teeth for intraoral, panoramic, and CBCT calculated by MC-GPU simulations are presented in Figure 2. The angles of radiation incidence to the head phantom used in the simulations (Table 1) are visible on the head anatomy: $30^{\circ}$ from the patient's front for intraoral; $240^{\circ}$ rotation behind the patient's head for panoramic; and $360^{\circ}$ rotation for CBCT. Movie clips presenting a rotating $3 \mathrm{D}$ dose distribution for panoramic and CBCT were created and included in the web dose calculator.

The fraction of dose in different tissues (brain, muscle, bone, skin, soft tissue, cerebrospinal, blood, and eye lens) out of the total dose for intraoral, panoramic, and CBCT is shown in Figure 3. A larger portion of the radiation dose is delivered to the bone (55\%) in intraoral imaging compared with panoramic and CBCT, each of which contributes about
$35 \%$ of the total radiation dose to the bone. The dose delivered to the brain is nearly zero in intraoral but slightly increased to $1 \%$ in panoramic and $2 \%$ in CBCT. The dose delivered to skin and soft tissue remarkably increases from $12 \%$ (soft tissue) and 10\% (skin) in intraoral to 26\% (soft tissue) and $12 \%$ (skin) in panoramic and 22\% (soft tissue) and $11 \%$ (skin) in CBCT.

3.4. Web Dose Calculator. A user-friendly interactive web program was developed for a user to input the following: the type of imaging procedure, the number of image sets, age group, and FOV size (Figure 4(a)). The web interface displays organ and effective doses (Figure 4(b)), dose fraction in tissues and $2 \mathrm{D}$ and $3 \mathrm{D}$ dose distributions in the head (Figure $4(\mathrm{c})$ ), and dose comparison with other radiation sources (Figure $4(\mathrm{~d})$ ).

\section{Discussion}

Dental imaging is one of the most common radiological imaging procedures. Although the dose level is known to be relatively low, it is still important to monitor the trend of dental dose in different dental imaging modalities. We evaluated the radiation dose received from dental imaging practices by extracting data from literature published after 2010. To efficiently present the results of the study, an interactive web-based dose calculator was created.

We compared our results from intraoral imaging with those published by Fontana et al. [32], which report the dose to the brain, salivary gland, and thyroid delivered by imaging conducted from 1940 to 2009, with the increment of ten years. To simplify the comparison, we averaged their doses in three time periods: 1940-1969, 1970-1989, and 1990-2009. The period 2010-2020 adopted in our study follows the end of their study period. A clear dose reduction was observed in the brain dose by period. Compared with the organ doses reported for the earliest period (1940-1969), the doses to the brain, salivary gland, and thyroid resulted from our study were smaller by $77 \%, 93 \%$, and $93 \%$, respectively. Compared with the latest period, 1990-2009, in Fontana et al., our organ doses were smaller by $7 \%, 64 \%$, and $62 \%$ for the brain, salivary gland, and thyroid, respectively. The dose reduction may be due to the change in technical parameters and the 
TABLE 6: Minimum, mean, and maximum values of effective and organ (bone marrow, brain, salivary gland, and thyroid) doses from conebeam computed tomography procedures by age group (children and adults) reported in the selected publications.

\begin{tabular}{ccccccc}
\hline \multirow{2}{*}{ Age group } & & \multirow{2}{*}{ Effective dose $(\mu \mathrm{Sv})$} & \multicolumn{3}{c}{ Organ dose $(\mu \mathrm{Gy})$} \\
& & & Bone marrow & Brain & Salivary gland & Thyroid \\
\hline \multirow{3}{*}{ Children $(n=22)$} & Mean & 143.9 & 372.2 & 600.7 & 2393.3 & 759.7 \\
& Min & 17.1 & 15.2 & 14.4 & 336.3 & 38.6 \\
& Max & 392.2 & 1478.0 & 4324.0 & 4352.0 & 2908.0 \\
\hline \multirow{3}{*}{ Adults $(n=54)$} & Mean & 111.8 & 207.0 & 419.1 & 2309.7 & 832.1 \\
& Min & 19.0 & 6.9 & 2.8 & 32.0 & 7775.0 \\
\hline
\end{tabular}

TABLE 7: Minimum, mean, and maximum values of effective and organ (bone marrow, brain, salivary gland, and thyroid) doses from conebeam computed tomography procedures by field-of-view area (FOV width $\times$ height, $\mathrm{cm}^{2}$ ) reported in the selected publications.

\begin{tabular}{ccccccc}
\hline \multirow{2}{*}{ FOV area $\left(\mathrm{cm}^{2}\right)$} & & \multirow{2}{*}{ Effective dose $(\mu \mathrm{Sv})$} & \multicolumn{3}{c}{ Organ dose $(\mu \mathrm{Gy})$} \\
& & & Bone marrow & Brain & Salivary gland & Thyroid \\
\hline \multirow{3}{*}{ Small $(<50)(n=20)$} & Mean & 96.5 & 262.8 & 144.2 & 1984.7 \\
& Min & 17.1 & 15.2 & 14.4 & 336.3 & 38.6 \\
& Max & 220.2 & 1052.0 & 760.0 & 4352.0 & 1252.0 \\
\hline \multirow{3}{*}{ Medium $(50-150)(n=32)$} & Mean & 113.7 & 246.4 & 184.9 & 2137.2 & 780.7 \\
& Min & 28.0 & 6.9 & 2.8 & 32.0 & 8.4 \\
& Max & 298.0 & 1478.0 & 1155.0 & 4700.0 & 3533.0 \\
\hline \multirow{3}{*}{ Large $(>150)(n=24)$} & Mean & 151.4 & 259.3 & 1126.8 & 2887.3 & 1267.7 \\
& Min & 31.0 & 10.0 & 124.0 & 738.3 & 47.0 \\
& Max & 392.2 & 1034.0 & 4653.0 & 7775.0 & 8727.0 \\
\hline
\end{tabular}

TABLE 8: Minimum, mean, and maximum values of effective and organ (bone marrow, brain, salivary gland, and thyroid) doses from conebeam computed tomography procedures by age group (children and adults) and field-of-view area (FOV width $\times$ height, $\mathrm{cm}^{2}$ ) reported in the selected publications.

\begin{tabular}{|c|c|c|c|c|c|c|c|}
\hline \multirow{2}{*}{\multicolumn{3}{|c|}{ FOV area $\left(\mathrm{cm}^{2}\right)$ age group }} & \multirow{2}{*}{ Effective dose $(\mu \mathrm{Sv})$} & \multicolumn{4}{|c|}{ Organ dose $(\mu \mathrm{Gy})$} \\
\hline & & & & Bone marrow & Brain & Salivary gland & Thyroid \\
\hline \multirow{6}{*}{ Small $(<50)$} & \multirow{3}{*}{ Children $(n=11)$} & Mean & 131.3 & 292.4 & 192.0 & 2568.5 & 281.4 \\
\hline & & Min & 17.1 & 15.2 & 14.4 & 336.3 & 38.6 \\
\hline & & Max & 220.2 & 1052.0 & 760.0 & 4352.0 & 751.0 \\
\hline & \multirow{3}{*}{ Adults $(n=9)$} & Mean & 53.8 & 226.6 & 85.8 & 1271.1 & 349.3 \\
\hline & & Min & 19.0 & 21.0 & 18.0 & 523.0 & 30.0 \\
\hline & & Max & 105.0 & 400.0 & 290.0 & 2830.0 & 1252.0 \\
\hline \multirow{6}{*}{ Medium (50-150) } & \multirow{3}{*}{ Children $(n=7)$} & Mean & 144.3 & 512.2 & 115.9 & 2289.2 & 1089.1 \\
\hline & & Min & 47.9 & 31.7 & 28.6 & 1028.0 & 270.6 \\
\hline & & Max & 298.0 & 1478.0 & 219.0 & 4204.0 & 2772.0 \\
\hline & \multirow{3}{*}{ Adults $(n=25)$} & Mean & 105.2 & 172.0 & 204.3 & 2094.7 & 694.4 \\
\hline & & Min & 28.0 & 6.9 & 2.8 & 32.0 & 8.4 \\
\hline & & Max & 265.0 & 621.0 & 1155.0 & 6372.0 & 3533.0 \\
\hline \multirow{6}{*}{ Large $(>150)$} & \multirow{3}{*}{ Children $(n=4)$} & Mean & 177.7 & 346.6 & 2572.9 & 2093.9 & 1498.6 \\
\hline & & Min & 64.7 & 104.7 & 772.2 & 1319.0 & 202.6 \\
\hline & & Max & 392.2 & 614.0 & 4324.0 & 4007.0 & 2908.0 \\
\hline & \multirow{3}{*}{ Adults $(n=20)$} & Mean & 146.1 & 241.9 & 837.6 & 3046.0 & 1221.5 \\
\hline & & Min & 31.0 & 10.0 & 124.0 & 738.3 & 47.0 \\
\hline & & Max & 368.0 & 1034.0 & 4653.0 & 7775.0 & 8727.0 \\
\hline
\end{tabular}

improvement in imaging quality with the same amount of radiation.

The average effective dose from CBCT, delivering the greatest dose compared with intraoral and panoramic, was more than 92 times greater than that from intraoral and seven times greater than that from panoramic (Table 4). However, the maximum CBCT effective dose, with a large
FOV, for children, $392.2 \mu \mathrm{Sv}$ [5], is about $13 \%$ of the dose from the natural radiation that a person receives on average every year, $3110 \mu \mathrm{Sv}$ [9], disregarding the radiation received from occupations and medical procedures.

We are aware of the following limitations in the current study. First, without dose calibration using measurements from clinical machines, absolute doses could not be 


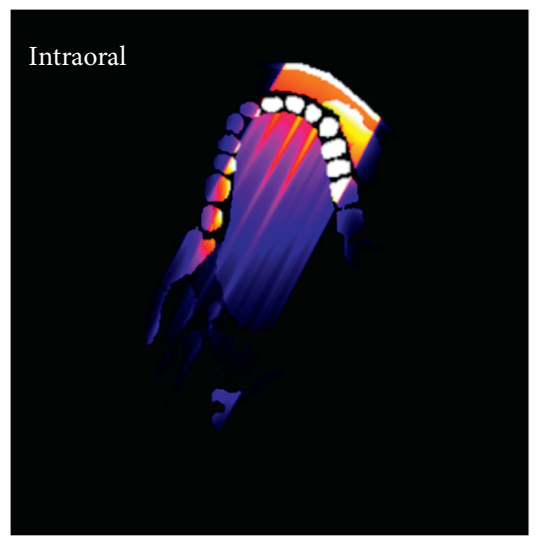

(a)

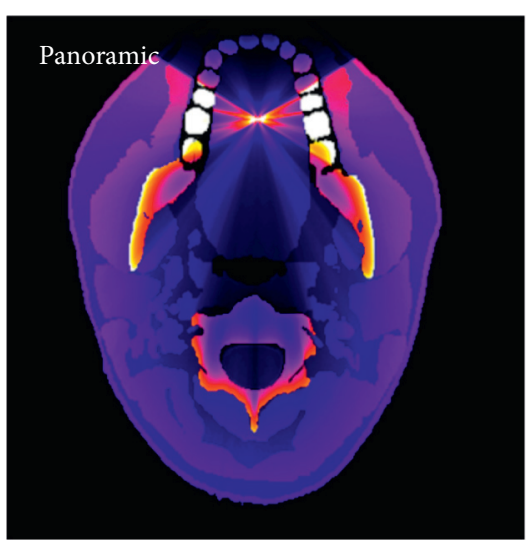

(b)

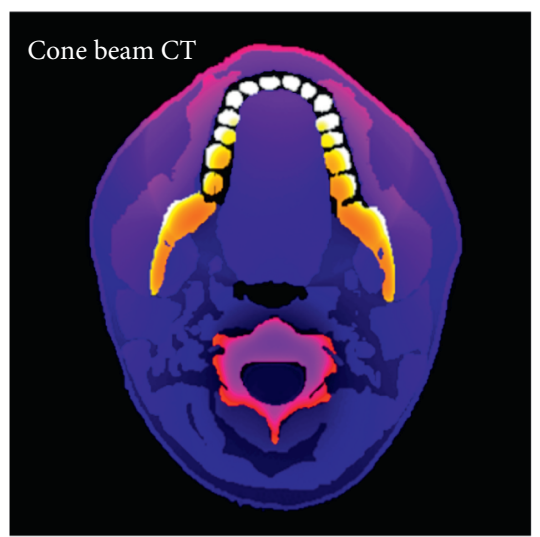

(c)

FIGURE 2: Dose distribution at the level of the lower teeth generated by MC-GPU simulations for (a) intraoral radiography, (b) panoramic radiography, and (c) cone-beam computed tomography.

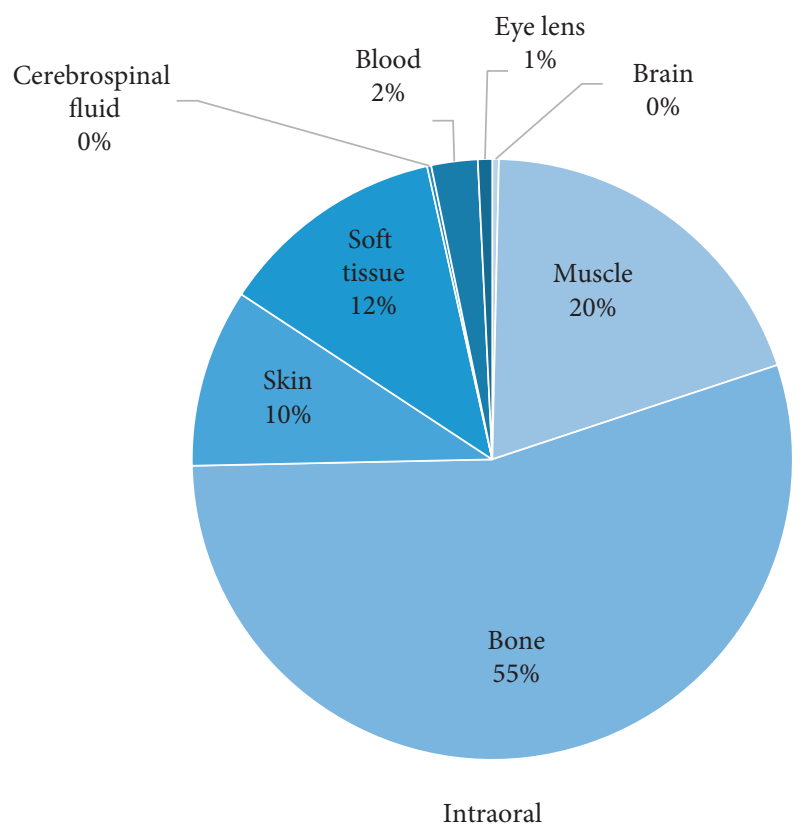

(a)

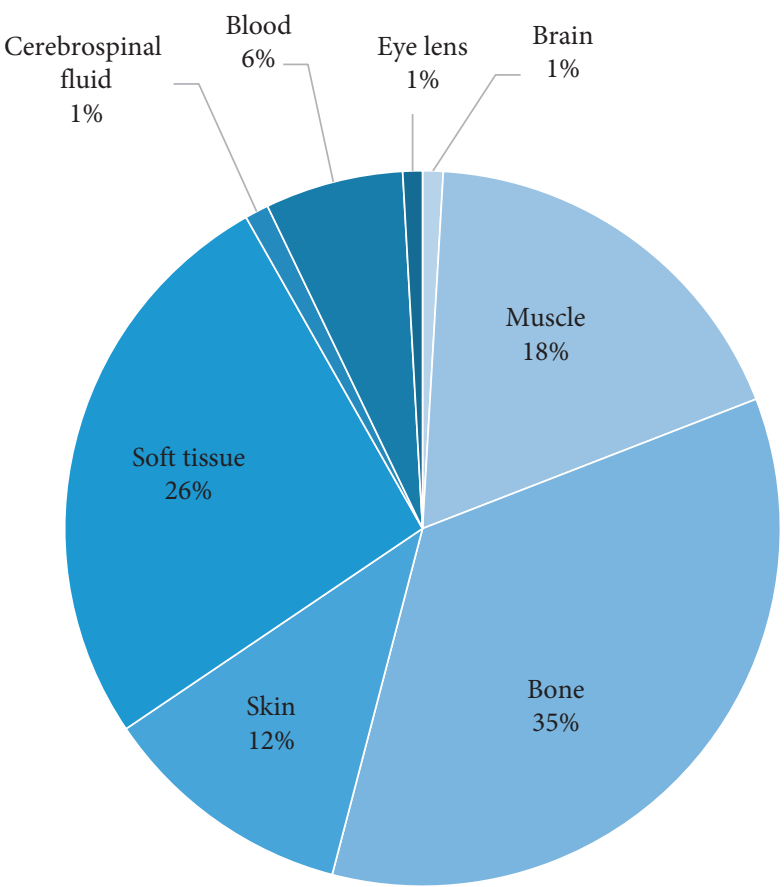

Panoramic

(b)

Figure 3: Continued. 


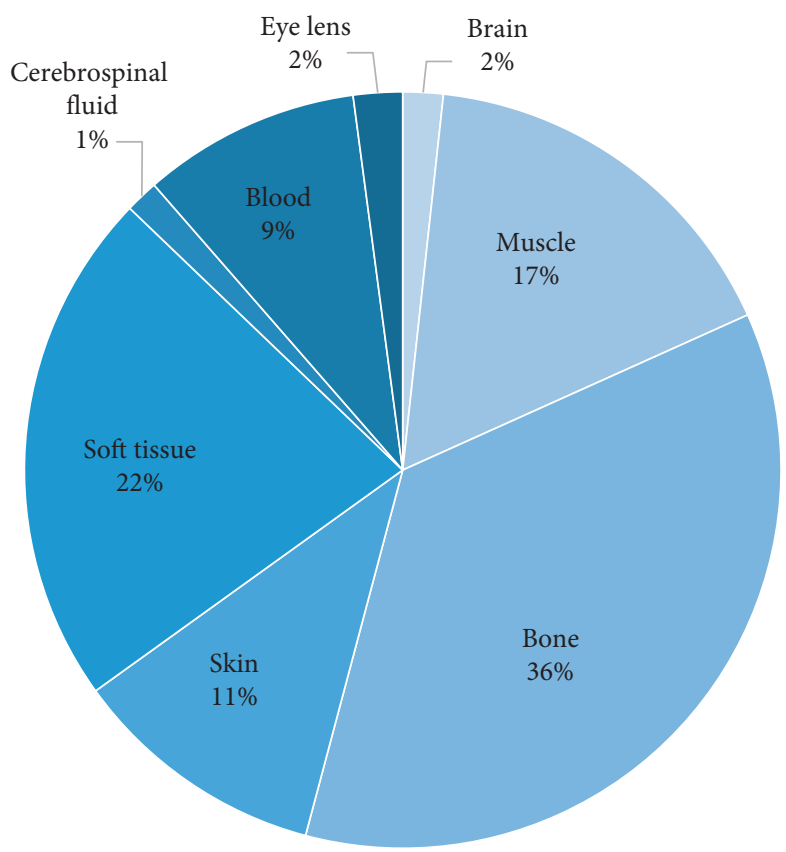

CBCT

(c)

Figure 3: Percent fraction of radiation dose for the different tissues in the head derived from the MC-GPU simulation of the human head phantom exposed to (a) intraoral radiography, (b) panoramic radiography, and (c) cone-beam computed tomography.

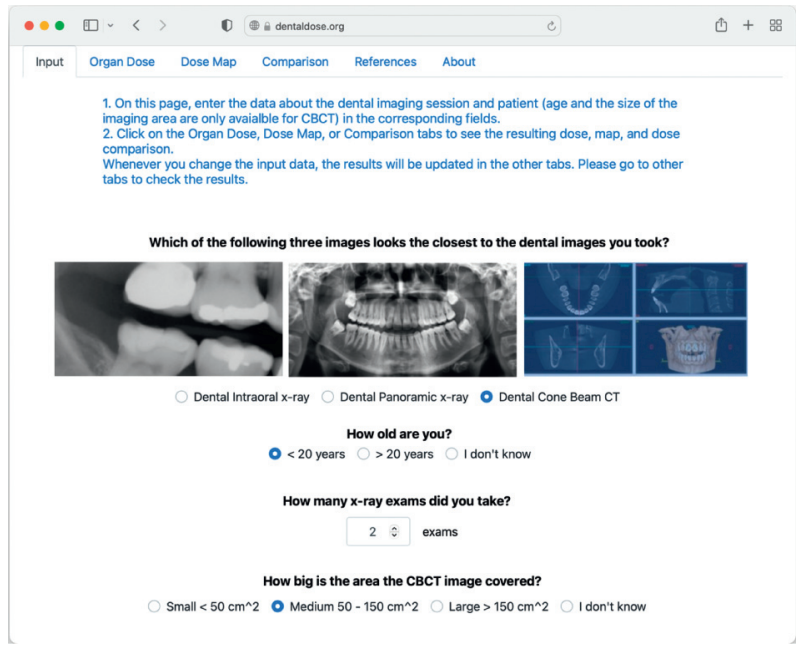

(a)

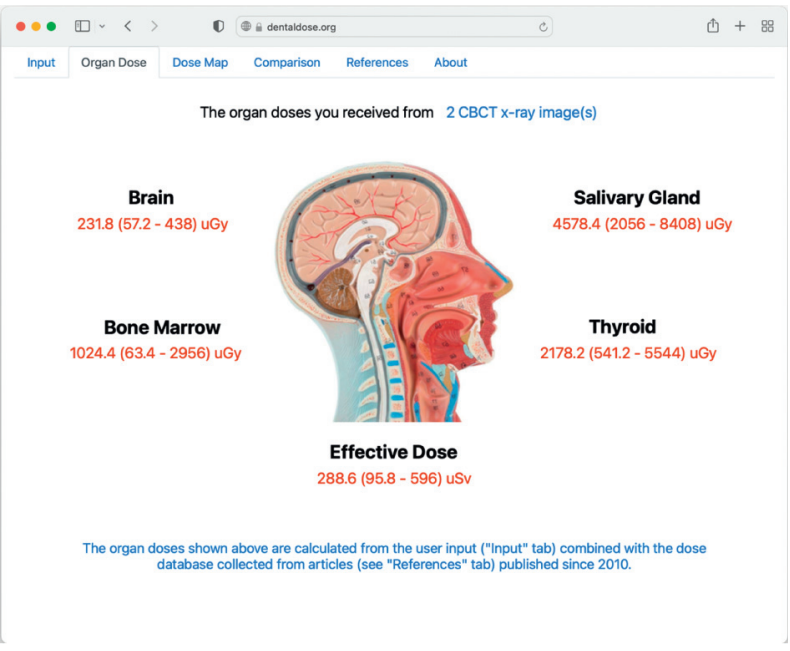

(b)

Figure 4: Continued. 


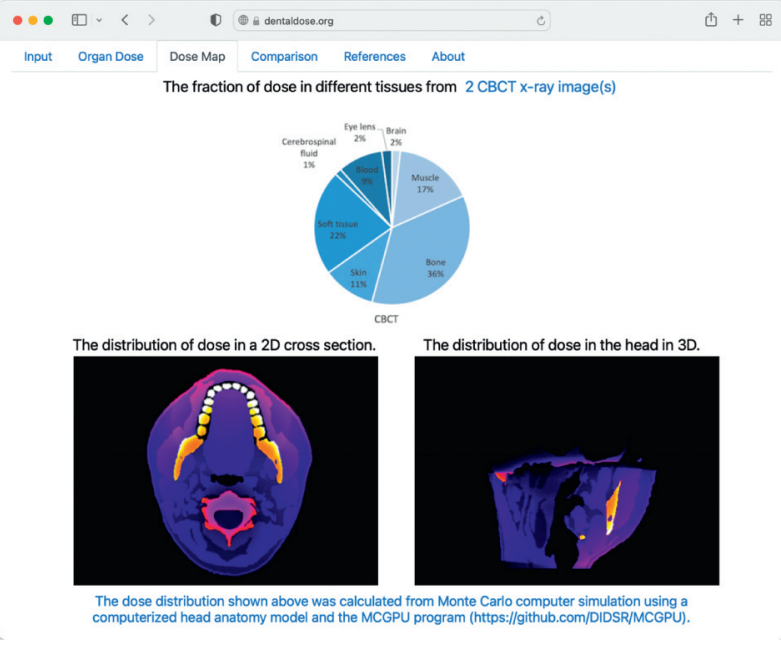

(c)

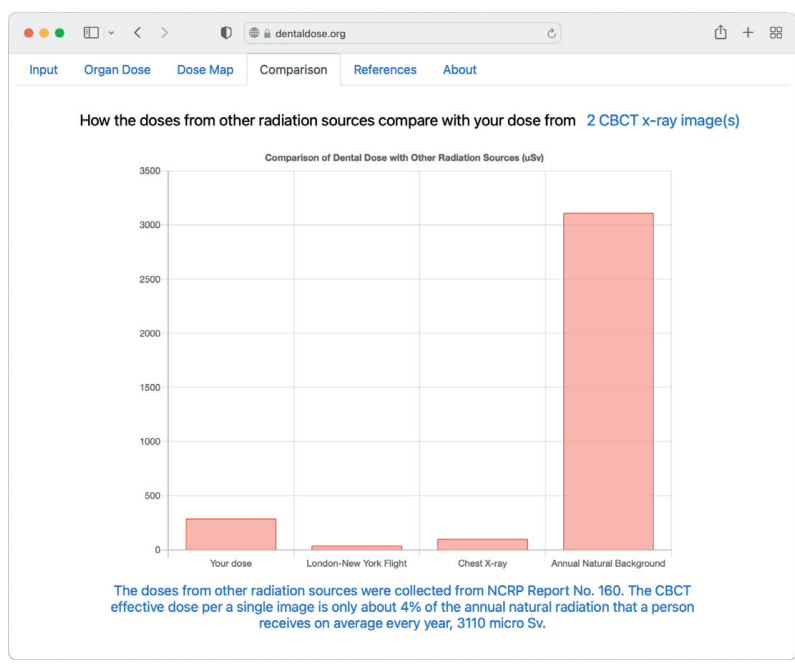

(d)

FIGURE 4: Web interfaces for (a) user input, (b) organ (brain, salivary gland, bone marrow, thyroid) and effective doses, (c) the fraction of dose in different tissues and 2D and 3D dose distributions, and (d) dose comparison with other radiation sources.

estimated with our Monte Carlo simulations, so only relative dose distributions were obtained and analyzed. Future work may involve accurate dose measurements to provide absolute doses for a comprehensive library of technical parameters for panoramic and CBCT procedures. Second, we found that pediatric dose data were relatively limited in literature compared with those of adults, so we grouped agedependent dose data into pediatric (age <20) and adult (age $\geq 20$ ) for CBCT only,for which a total of 22 pediatric data points were available. Since a clear age dependency for those limited data points was not observed, possibly due to large variability, we categorized the pediatric ages into a single group. Considering the higher potential risk in pediatric patients, due to increased radiosensitivity and longer expected life span after the irradiation event, it is important to more accurately evaluate the doses delivered to them once additional dose data are available in the future. Lastly, our literature search was limited to one bibliographic database, PubMed, to the keywords we defined, and to the papers written in English.

\section{Conclusion}

A comprehensive table of the organ and effectives doses delivered by intraoral, panoramic, and CBCT dental imaging procedures was established from previously published articles collected from PubMed. We found that organ and effective doses from intraoral and panoramic radiography are substantially smaller than those from $\mathrm{CBCT}$, and the maximum CBCT effective dose is about $13 \%$ of the dose from annual natural radiation. Our dose summary should be useful for comparison among doses from different dental imaging methods as well as comparison with doses from other radiation sources. The user-friendly, interactive web application (http://dentaldose.org) allows for receiving user input and displaying doses, dose distribution maps, and dose comparison with other radiation sources.

\section{Data Availability}

The radiation dose data from dental imaging procedures used to support the findings of this study are included within the article.

\section{Conflicts of Interest}

The authors declare that they have no conflicts of interest.

\section{Acknowledgments}

This work was funded by the 2020 Oak Ridge Institute for Science and Education (ORISE) Research Fellowship Program at the US Food and Drug Administration.

\section{References}

[1] Ö Özalp, H. A. Tezerişener, B. Kocabalkan et al., "Comparing the precision of panoramic radiography and cone-beam computed tomography in avoiding anatomical structures critical to dental implant surgery: a retrospective study," Imaging Science in Dentistry, vol. 48, pp. 269-275, 2018.

[2] A. Stratis, G. Zhang, R. Jacobs, R. Bogaerts, and H. Bosmans, "The growing concern of radiation dose in paediatric dental and maxillofacial CBCT: an easy guide for daily practice," European Radiology, vol. 29, no. 12, pp. 7009-7018, 2019.

[3] E. Pakbaznejad Esmaeili, M. Ekholm, J. Haukka, M. Evälahti, and J. Waltimo-Sirén, “Are children's dental panoramic tomographs and lateral cephalometric radiographs sufficiently optimized?" The European Journal of Orthodontics, vol. 38, no. 1, pp. 103-110, 2016.

[4] ICRP, The 2007 Recommendations of the International Commission on Radiological Protection, ICRP Publication 103, Ann, ICRP 37, 2007.

[5] X. G. Xu, "An exponential growth of computational phantom research in radiation protection, imaging, and radiotherapy: a review of the fifty-year history," Physics in Medicine and Biology, vol. 59, no. 18, pp. R233-R302, 2014. 
[6] ICRP, Basic Anatomical and Physiological Data for Use in Radiological Protection Reference Values, ICRP Publication 89, Ann, ICRP 32, 2002.

[7] M. I. Iacono, E. Neufeld, E. Akinnagbe et al., "MIDA: a multimodal imaging-based detailed anatomical model of the human head and neck," PLoS One, vol. 10, no. 4, p. e0124126, 2015.

[8] A. Badal and A. Badano, "Accelerating Monte Carlo simulations of photon transport in a voxelized geometry using a massively parallel graphics processing unit," Medical Physics, vol. 36, no. 11, pp. 4878-4880, 2009.

[9] N. C. RP. Ionizing, Radiation Exposure of the Population of the United States, National Council on Radiation Protection and Measurement, Bethesda, MD, USA, 2009.

[10] C. Granlund, A. Thilander-Klang, B. Ylhan, S. LofthagHansen, and A. Ekestubbe, "Absorbed organ and effective doses from digital intra-oral and panoramic radiography applying the ICRP 103 recommendations for effective dose estimations," The British Journal of Radiology, vol. 89, no. 1066, p. 20151052, 2016.

[11] N. Kadesjö, R. Lynds, M. Nilsson, and X. Q. Shi, "Radiation dose from X-ray examinations of impacted canines: cone beam CT vs two-dimensional imaging," Dento Maxillo Facial Radiology, vol. 47, p. 20170305, 2018.

[12] Y. Li, B. Huang, J. Cao et al., "Estimating radiation dose to major organs IN dental X-ray examinations: a phantom study," Radiation Protection Dosimetry, vol. 192, no. 3, pp. 328-334, 2020.

[13] A. Matsuo, T. Okano, K. Gotoh et al., "The absorbed dose and the effective dose of panoramic temporo mandibular joint radiography," Japanese Journal of Radiological Technology, vol. 67, no. 10, pp. 1275-1283, 2011.

[14] G.-S. Han, J.-G. Cheng, G. Li, and X.-C. Ma, "Shielding effect of thyroid collar for digital panoramic radiography," Dentomaxillofacial Radiology, vol. 42, no. 9, p. 20130265, 2013.

[15] A. T. Davis, H. Safi, and S. M. Maddison, "The reduction of dose in paediatric panoramic radiography: the impact of collimator height and programme selection," Dentomaxillofacial Radiology, vol. 44, no. 2, p. 20140223, 2015.

[16] C. Lee, S.-S. Lee, J.-E. Kim et al., "Comparison of dosimetry methods for panoramic radiography: thermoluminescent dosimeter measurement versus personal computer-based Monte Carlo method calculation," Oral Surgery, Oral Medicine, Oral Pathology and Oral Radiology, vol. 121, no. 3, pp. 322-329, 2016.

[17] W. Qiang, F. Qiang, and L. Lin, "Estimation of effective dose OF dental X-ray devices," Radiation Protection Dosimetry, vol. 183, pp. 417-421, 2019.

[18] C. Lee, B. Park, S.-S. Lee et al., "Efficacy of the Monte Carlo method and dose reduction strategies in paediatric panoramic radiography," Scientific Reports, vol. 9, no. 1, p. 9691, 2019.

[19] R. Pauwels, J. Beinsberger, B. Collaert et al., "Effective dose range for dental cone beam computed tomography scanners," European Journal of Radiology, vol. 81, no. 2, pp. 267-271, 2012.

[20] J. Koivisto, T. Kiljunen, M. Tapiovaara, J. Wolff, and M. Kortesniemi, "Assessment of radiation exposure in dental cone-beam computerized tomography with the use of metaloxide semiconductor field-effect transistor (MOSFET) dosimeters and Monte Carlo simulations," Oral Surgery, Oral Medicine, Oral Pathology and Oral Radiology, vol. 114, no. 3, pp. 393-400, 2012.

[21] J. Morant, M. Salvadó, I. Hernández-Girón, R. Casanovas, R. Ortega, and A. Calzado, "Dosimetry of a cone beam CT device for oral and maxillofacial radiology using Monte Carlo techniques and ICRP adult reference computational phantoms," Dentomaxillofacial Radiology, vol. 42, no. 3, p. $92555893,2013$.

[22] A. Stratis, G. Zhang, R. Jacobs, R. Bogaerts, and H. Bosmans, "Rotating and translating anthropomorphic head voxel models to establish an horizontal Frankfort plane for dental CBCT Monte Carlo simulations: a dose comparison study," Physics in Medicine and Biology, vol. 61, no. 24, pp. N681N696, 2016.

[23] J.-K. Yeh and C.-H. Chen, "Estimated radiation risk of cancer from dental cone-beam computed tomography imaging in orthodontics patients," BMC Oral Health, vol. 18, no. 1, p. 131, 2018.

[24] M. Marcu, M. Hedesiu, B. Salmon et al., "Estimation of the radiation dose for pediatric $\mathrm{CBCT}$ indications: a prospective study on ProMax3D," International Journal of Paediatric Dentistry, vol. 28, no. 3, pp. 300-309, 2018.

[25] I. Kralik, D. Faj, T. Lauc, M. Škarica, J. Popić, and H. Brkic, "Dose area product in estimation of effective dose of the patients undergoing dental cone beam computed tomography examinations," Journal of Radiological Protection, vol. 38, no. 4, pp. 1412-1427, 2018.

[26] C. Lee, J. Yoon, S.-S. Han et al., "Dose assessment in dental cone-beam computed tomography: comparison of optically stimulated luminescence dosimetry with Monte Carlo method," PLoS One, vol. 15, no. 3, p. e0219103, 2020.

[27] I. Kawrakow and D. W. O. Rogers, The EGSnrc Code System: Monte Carlo Simulation of Electron and Photon Transport, National Research Council of Canada, Ottawa, Canada, 2003.

[28] J. F. Briesmeister, MCNP - A General Monte Carlo N-Particle Transport Code, Los Alamos National Laboratory, Los Alamos, NM, USA, 1997.

[29] M. Tapiovaara, M. Lakkisto, and A. Servomaa, PCXMC-A PCBased Monte Carlo Program for Calculating Patient Doses in Medical X-Ray Examinations, Radiation and Nuclear Safety Authority (STUK), Helsinki, Finland, 2nd edition, 2008.

[30] I. G. Zubal, C. R. Harrell, E. O. Smith, Z. Rattner, G. Gindi, and P. B. Hoffer, "Computerized three-dimensional segmented human anatomy," Medical Physics, vol. 21, no. 2, pp. 299-302, 1994.

[31] ICRP, "Adult reference computational phantoms. ICRP publication 110," ICRP Publication, ICRP 39, 2, 2009.

[32] R. C. Fontana, E. Pasqual, D. L. Miller, S. L. Simon, E. Cardis, and I. Thierry-Chef, "Trends in estimated thyroid, salivary gland, brain, and eye lens doses from intraoral dental radiography over seven decades (1940 TO 2009)," Health Physics, vol. 118, no. 2, pp. 136-148, 2020. 